\title{
Steady-state, relaxation and first-passage properties of a run-and-tumble particle in one-dimension
}

\author{
Kanaya Malakar ${ }^{1}$, V. Jemseena ${ }^{2}$, Anupam Kundu ${ }^{2}$, K. Vijay \\ Kumar $^{2}$, Sanjib Sabhapandit ${ }^{3}$, Satya N. Majumdar ${ }^{4}$, S. \\ Redner $^{5}$, Abhishek Dhar ${ }^{2}$ \\ ${ }^{1}$ Presidency University, 86/1, College Street, Kolkata 700073, India \\ ${ }^{2}$ International Centre for Theoretical Sciences, Tata Institute of Fundamental \\ Research, Bengaluru 560089, India \\ ${ }^{3}$ Raman Research Institute, Bangalore 560080, India \\ ${ }^{4}$ LPTMS, CNRS, Univ. Paris-Sud, Université Paris-Saclay, 91405 Orsay, France \\ ${ }^{5}$ Santa Fe Institute, 1399 Hyde Park Road, Santa Fe, New Mexico 87501, USA
}

\begin{abstract}
We investigate the motion of a run-and-tumble particle (RTP) in one dimension. We find the exact probability distribution of the particle with and without diffusion on the infinite line, as well as in a finite interval. In the infinite domain, this probability distribution approaches a Gaussian form in the long-time limit, as in the case of a regular Brownian particle. At intermediate times, this distribution exhibits unexpected multi-modal forms. In a finite domain, the probability distribution reaches a steady-state form with peaks at the boundaries, in contrast to a Brownian particle. We also study the relaxation to the steady-state analytically. Finally we compute the survival probability of the RTP in a semi-infinite domain with an absorbing boundary condition at the origin. In the finite interval, we compute the exit probability and the associated exit times. We provide numerical verification of our analytical results.
\end{abstract}

\section{Introduction}

Active particles are self-driven systems, where the dynamics has a dissipative and a stochastic part. Their dynamics violates fluctuation-dissipation relation. This system naturally breaks detailed balance and has been widely used to understand various nonequilibrium phenomena which are driven at the level of individual constituents, for example motion of bacteria, flocking of birds and vibrated granular matter [1 6$]$. Runand-tumble particles (RTPs) and active Brownian particles (ABPs) are the simplest examples of such active particles and are known to exhibit interesting features such as non-Boltzmann distributions in the steady-state [7 17], clustering [18, 19], spontaneous segregation of mixtures of active and passive particles [20, ratchet effects 21] and motility-induced phase separation 22 25]. Recent studies show that, unlike equilibrium systems, these systems may not have an equation of state for the mechanical pressure 26 28]. 
The stochastic dynamics used to describe the motion of RTPs and other active particles has been studied earlier in the context of systems with colored noise, and some exact as well as approximate analytic results for steady-states and first-passage properties were obtained [29,30]. The dynamics of active particles is related to the equilibrium properties of semi-flexible polymers, where many analytic results are known [31,32]. There have been recent attempts to understand the time evolution of the probability distributions of active particles in unbounded geometries [33]. In confined geometries, RTPs and ABPs are known to accumulate near the boundaries of the domain [7]. The steady-state distribution of such active particles in confining potentials are non-Boltzmannian [30,34-36] and can exhibit jammed states [18,37]. More recently there have been a number of studies on computing the steady-state distribution for both RTPs and ABPs in various confined geometries, but using approximate methods in most cases 38 41]. However, so far, the approach to the steady-state has not been studied in detail.

Given the rich behavior of RTPs, it is worthwhile to study them in the simplest possible setting where we can derive explicit results for basic dynamical observables. In this spirit, we investigate the dynamics of non-interacting RTPs with an additional Brownian diffusion term. We investigate the motion on: (i) the infinite line, (ii) a one-dimensional bounded domain with reflecting walls, and (iii) the semi-infinite line and the bounded domain with absorbing boundaries. The restriction to one dimension greatly simplifies the analysis, without sacrificing phenomenological richness.

We implement run and tumble motion by imposing a particle velocity $v$ that switches sign at a random Poisson rate. Naively, one might anticipate that this velocity switching merely renormalizes the diffusion coefficient. Such an interplay between advection and diffusion underlies, for example, the phenomenon of hydrodynamic dispersion 42 45. Here, a diffusing tracer is passively carried by a flow field, such as Poiseuille flow in a pipe, and the combination of microscopic diffusion and convection leads to a greatly enhanced spread of the tracer in the longitudinal direction. A similar phenomenon arises for RTPs in the unbounded geometry in the long-time limit. However, there are surprising pre-asymptotic effects. For a wide range of parameters, the probability distribution evolves from unimodal, to multimodal, before finally converging to a Gaussian in the long-time limit. We also compute the steady-state of a RTP inside a finite domain, and examine at the approach to the steady-state. The approach to the steady-state is studied by examining the spectral structure of the relevant FokkerPlanck operator, and we find that this problem is highly non-trivial. Finally we study first-passage properties of the RTP inside a semi-infinite domain where we obtain exact analytic results for the first-passage distribution and exit time probabilities. We compare these results with the usual diffusive case and point out the qualitative differences.

This paper is organized as follows. In section 2, we define the model and discuss the relevant boundary conditions for the probability distributions in a finite interval. In section 3.1, we calculate the propagators for RTPs with superimposed diffusion in an unbounded domain and thereby derive the exact probability distribution. We 
study RTPs in a bounded domain and calculate their steady-state and time-dependent distributions in section 3.2. Finally we turn to first-passage properties of an RTP where we calculate its survival probability in a semi-infinite one-dimensional domain with absorbing walls (Sec. 4.1) and the exit times in this domain (Sec. 4.2). Throughout this work, we compare our exact results with numerical simulations of the Langevin equations for the RTPs and numerical solutions of the associated Fokker-Planck equation.

\section{RTP Model}

We study a particle that moves on the one-dimensional line whose motion is described by the following stochastic equation

$$
\frac{d x}{d t}=v \sigma(t)+\sqrt{2 D} \eta(t)
$$

where the random variable $\sigma(t)$ switches between \pm 1 at a Poisson rate $\gamma$, and $\eta(t)$ is Gaussian white noise with

$$
\langle\eta(t)\rangle=0, \quad\left\langle\eta(t) \eta\left(t^{\prime}\right)\right\rangle=\delta\left(t-t^{\prime}\right) .
$$

Equation (1) can be reduced to a Markovian model if we specify the particle state by both its position $x$ and its current velocity $( \pm 1)$. It is convenient to define $P_{+}(x, t)$ and $P_{-}(x, t)$ as the probability density for the particle to be at position $x$ with velocities $+v$ and $-v$, respectively. These state probabilities evolve according to the generalized form of telegrapher's equation

$$
\begin{aligned}
& \partial_{t} P_{+}=D \partial_{x}^{2} P_{+}-v \partial_{x} P_{+}-\gamma P_{+}+\gamma P_{-}, \\
& \partial_{t} P_{-}=D \partial_{x}^{2} P_{-}+v \partial_{x} P_{-}+\gamma P_{+}-\gamma P_{-} .
\end{aligned}
$$

This equation was perhaps derived first in the context of electromagnetic theory 46] and was later derived in several other contexts (see the review [47] and the references therein). The probability $P(x, t)$ to find the particle at position $x$ at time $t$ is the sum of the probabilities $P_{ \pm}(x, t)$ of finding the particle in the two states, i.e., $P=P_{+}+P_{-}$. We choose $\gamma^{-1}$ as the unit of time and $v \gamma^{-1}$ as the unit of length to recast (3a) in the dimensionless form

$$
\begin{aligned}
& \partial_{t} P_{+}=\mathcal{D} \partial_{x}^{2} P_{+}-\partial_{x} P_{+}-P_{+}+P_{-}, \\
& \partial_{t} P_{-}=\mathcal{D} \partial_{x}^{2} P_{-}+\partial_{x} P_{-}+P_{+}-P_{-},
\end{aligned}
$$

where the dimensionless diffusion constant $\mathcal{D}=D \gamma / v^{2}$ is the only parameter for the unbounded system.

For the finite interval $[-L, L]$, there is a second parameter: the dimensionless interval length $\ell=L \gamma / v$. When the particle is restricted to a finite domain $x \in[-\ell, \ell]$, we impose the boundary condition that when the particle hits the boundary, it stays stuck there until its internal state $( \pm)$ changes, upon which it can move away from the boundary. Hence there is no particle current across these walls. From Eqs. $(3 \mathrm{~b})$, we identify the particle currents $J_{ \pm}(x, t)$ at position $x$ and time $t$ as:

$$
J_{ \pm}(x, t)=-\mathcal{D} \partial_{x} P_{ \pm} \pm P_{ \pm} .
$$


The following four boundary conditions are obtained by demanding that the value of these currents is zero at $x= \pm \ell$, that is,

$$
\begin{gathered}
\left(\mathcal{D} \partial_{x} P_{+}-P_{+}\right)_{x= \pm \ell}=0 \\
\left(\mathcal{D} \partial_{x} P_{-}+P_{-}\right)_{x= \pm \ell}=0 .
\end{gathered}
$$

\section{The Occupation Probability $P(x, t)=P_{+}(x, t)+P_{-}(x, t)$ :}

We now determine the RTP occupation probability $P(x, t)$, namely, the probability that the particle is at position $x$ at time $t$ for: (a) the infinite line and (b) the finite interval $[-\ell, \ell]$. To compute $P(x, t)$, we need to solve the coupled Fokker-Planck equations 3b for $P_{ \pm}(x, t)$ with the appropriate boundary conditions.

\subsection{Infinite domain: $\ell=\infty$}

It is useful to define the Fourier transforms $\tilde{P}_{ \pm}(k)=\int_{-\infty}^{\infty} P_{ \pm}(x, t) e^{i k x} d x$. Fourier transforming Eqs. (3b) with respect to $x$, we obtain (in matrix form):

$$
\frac{d}{d t}\left(\begin{array}{l}
\tilde{P}_{+}(k, t) \\
\tilde{P}_{-}(k, t)
\end{array}\right)=\mathbb{A}_{k}\left(\begin{array}{l}
\tilde{P}_{+}(k, t) \\
\tilde{P}_{-}(k, t)
\end{array}\right)
$$

where

$$
\mathbb{A}_{k}=\left(\begin{array}{cc}
-1-i k-\mathcal{D} k^{2} & \gamma \\
\gamma & -1+i k-\mathcal{D} k^{2}
\end{array}\right)
$$

Diagonalizing the matrix $\mathbb{A}_{k}$ for each $k$ and solving the resulting linear equations gives

$$
\left(\begin{array}{c}
\tilde{P}_{+}(k, t) \\
\tilde{P}_{-}(k, t)
\end{array}\right)=\mathbb{W}_{k}\left(\begin{array}{c}
\tilde{P}_{+}(k, 0) \\
\tilde{P}_{-}(k, 0)
\end{array}\right)
$$

where

$$
\mathbb{W}_{k}=\left(\begin{array}{cc}
\frac{e^{\alpha_{+} t}}{\mathcal{N}_{+}^{2}}+\frac{e^{\alpha_{-} t}}{\mathcal{N}_{+}^{2}}\left(i k+\sqrt{1-k^{2}}\right)^{2} & \frac{\gamma\left(e^{\alpha_{+} t}-e^{\alpha_{-} t}\right)}{2 \sqrt{1-k^{2}}} \\
\frac{\gamma\left(e^{\alpha_{+} t}-e^{\alpha_{-}}\right)}{2 \sqrt{1-k^{2}}} & \frac{e^{\alpha_{+} t}}{\mathcal{N}_{-}^{2}}+\frac{e^{\alpha_{-} t}}{\mathcal{N}_{-}^{2}}\left(i k-\sqrt{1-k^{2}}\right)^{2}
\end{array}\right)
$$

with $\mathcal{N}_{ \pm}=\sqrt{2}\left(1-k^{2} \pm i k \sqrt{1-k^{2}}\right)^{1 / 2}$ and $\alpha_{ \pm}=-\left(1+\mathcal{D} k^{2}\right) \pm \sqrt{1-k^{2}}$.

Consider the natural initial condition in which the particle starts at $x=0$, with equal probability to be either in the + or the - state. The Fourier transform of the initial probability is then $\tilde{P}(k, 0)=(4 \pi)^{-1}$. Using this in (7) and simplifying, we find

$$
\tilde{P}_{ \pm}(k, t)=\frac{e^{\alpha_{+} t}}{2}\left(\frac{1}{\mathcal{N}_{ \pm}^{2}}+\frac{1}{2 \sqrt{1-k^{2}}}\right)+\frac{e^{\alpha_{-} t}}{2}\left(\frac{\left(i k+\sqrt{1-k^{2}}\right)^{2}}{\mathcal{N}_{ \pm}^{2}}-\frac{1}{2 \sqrt{1-k^{2}}}\right) .
$$

From (8), the Fourier transform of the total probability $\tilde{P}(k, t)=\tilde{P}_{+}(k, t)+\tilde{P}_{-}(k, t)$ is

$$
\tilde{P}(k, t)=e^{-\left(1+\mathcal{D} k^{2}\right) t}\left[\cosh \left(t \sqrt{1-k^{2}}\right)+\frac{1}{\sqrt{1-k^{2}}} \sinh \left(t \sqrt{1-k^{2}}\right)\right] .
$$


We can alternatively derive this result as follows: The displacement of an RTP that starts at $x=0$, can be written formally by integrating the Langevin equation (1) to give $x(t)=\int_{0}^{t} \sigma(t) d t+\sqrt{2 \mathcal{D}} \int_{0}^{t} \xi(t) d t \equiv A(t)+B(t)$. Since the random processes $A(t)$ and $B(t)$ are independent of each other, $\tilde{P}(k, t)=\left\langle e^{i k A(t)}\right\rangle\left\langle e^{i k B(t)}\right\rangle$. It is easy to see that the expression in $(9)$ is actually in this product form once one identifies $\left\langle e^{i k B(t)}\right\rangle=e^{-\mathcal{D} k^{2} t}$ for the Brownian motion $B(t)$. The process $A(t)$ is the motion of an RTP with $\mathcal{D}=0$, whose dynamics is described by the telegrapher's equation, for which $\left\langle e^{i k A(t)}\right\rangle$ can be computed explicitly (see e.g., [31,32]). This immediately leads to the expression in (9).

Using the product structure of $\tilde{P}(k, t)$, we invert the Fourier transform in (9) to derive the probability $P(x, t)$ in the convolution form $P(x, t)=\int_{-\infty}^{\infty} g(x-y, t) h(y, t)$ where $g(x, t)=\exp \left(-x^{2} / 4 \mathcal{D} t\right) / \sqrt{4 \pi \mathcal{D} t}$ is the inverse Fourier transform of $\left\langle e^{i k B(t)}\right\rangle=$ $e^{-\mathcal{D} k^{2} t}$ and $h(x, t)$ is the inverse Fourier transform of $\left\langle e^{i k A(t)}\right\rangle$. Using the explicit expression of $h(x, t)$ from 31, 32, we obtain

$$
\begin{aligned}
P(x, t) & =\frac{\cosh (x / 2 \mathcal{D})}{\sqrt{4 \pi \mathcal{D} t}} e^{-t-\left(x^{2}+t^{2}\right) / 4 \mathcal{D} t} \\
& +\frac{e^{-t}}{2} \int_{-\infty}^{\infty} d y \frac{e^{-(x-y)^{2} / 4 \mathcal{D} t}}{\sqrt{4 \pi \mathcal{D} t}}\left[I_{0}\left(\sqrt{t^{2}-y^{2}}\right)+\frac{t}{\sqrt{t^{2}-y^{2}}} I_{1}\left(\sqrt{t^{2}-y^{2}}\right)\right] \Theta(t-|y|),
\end{aligned}
$$

where $I_{n}$ is the $n^{\text {th }}$-order modified Bessel function of the first kind and $\Theta$ is the Heaviside step function. Note that in the limit $x, t \rightarrow \infty, P(x, t)$ reduces to a simple Gaussian with diffusion constant $(\mathcal{D}+1 / 2)$. This can be easily seen from (9) where $\tilde{P}(k, t) \rightarrow \exp \left[-(\mathcal{D}+1 / 2) k^{2} t\right]$ as $t \rightarrow \infty$ and $k \rightarrow 0$.

It is instructive to examine the spatial moments of the probability distribution. All odd moments are zero by symmetry. Formally, the even moments of the distribution are given by

$$
\left\langle x^{2 n}(t)\right\rangle=\left.(-1)^{n} \frac{\partial^{2 n} \tilde{P}(k, t)}{\partial k^{2 n}}\right|_{k=0} .
$$

For the second moment, we find

$$
\left\langle x^{2}(t)\right\rangle=(2 \mathcal{D}+1) t-\frac{\left(1-e^{-2 t}\right)}{2} \rightarrow\left(2 D+v^{2} / \gamma\right) t-\frac{v^{2}}{2 \gamma^{2}}\left(1-e^{-2 \gamma t}\right) .
$$

where in the last simplification we have put in all the dimensional parameters. The above result has two non-trivial limiting cases. For $\gamma \neq 0$ and $t \rightarrow \infty$, 11) reduces to

$$
\left\langle x^{2}(t)\right\rangle \simeq(2 \mathcal{D}+1) t \rightarrow\left(2 D+v^{2} / \gamma\right) t
$$

In the $t \rightarrow \infty$ limit, the finite switching rate $\gamma$ leads to an enhancement of the microscopic diffusion coefficient in a manner that is reminiscent of hydrodynamic dispersion $42-45]$. On the other hand, in the limit $\gamma \rightarrow 0$, we find

$$
\left\langle x^{2}(t)\right\rangle \rightarrow 2 \mathcal{D} t+v^{2} t^{2}
$$



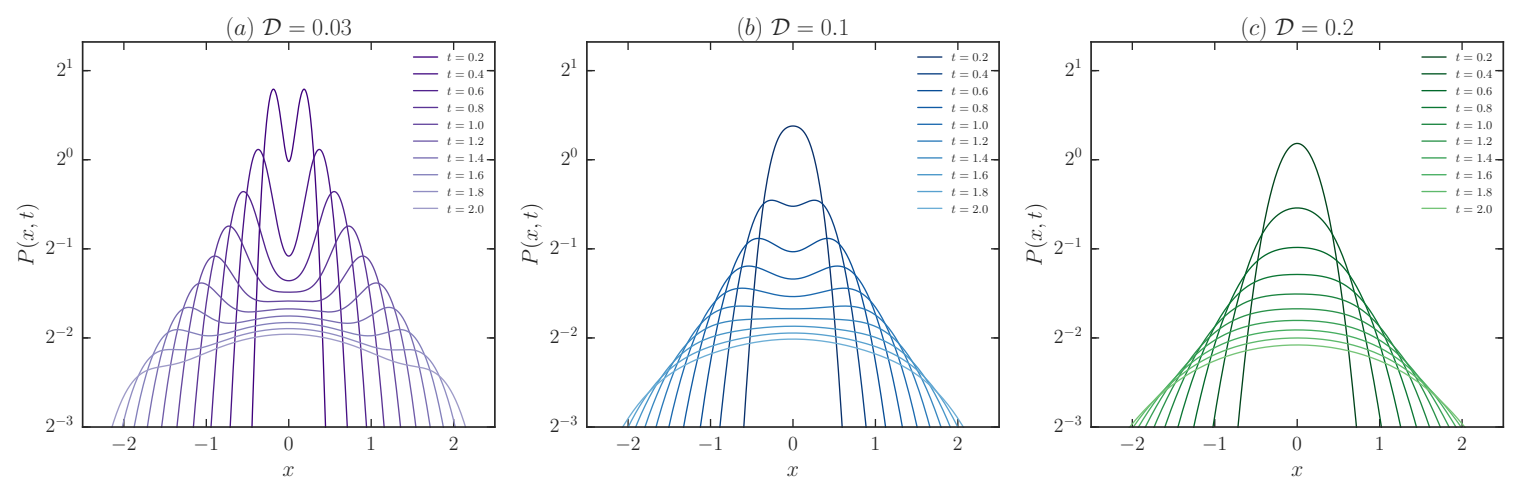

Figure 1. Plot of the probability density $P(x, t)$ in $(10)$ for three different values of the diffusion constant $\mathcal{D}$.

Thus the mean-square displacement crosses over from growing linearly with $t$ to quadratically with $t$ as $\gamma \rightarrow 0$.

We can also compute higher-order derivatives of $\tilde{P}(k, t)$ from which higher moments of the displacement can be deduced. The fourth moment is

$$
\left\langle x^{4}(t)\right\rangle=3 t^{2}\left(2 D+\frac{v^{2}}{\gamma}\right)^{2}-\frac{3 v^{2} t}{\gamma^{3}}\left[2 D \gamma\left(1-e^{-2 \gamma t}\right)-v^{2}\left(2+e^{-2 \gamma t}\right)\right]+\frac{9 v^{2}}{2 \gamma^{4}}\left(1-e^{-2 \gamma t}\right) .
$$

The important feature of this last result is that as $t \rightarrow \infty,\left\langle x^{4}\right\rangle / 3\left\langle x^{2}\right\rangle^{2} \rightarrow 1$, which is just the relation between the fourth and second moments for a Gaussian distribution. The behavior of the higher moments also conforms to those of the Gaussian distribution as $t \rightarrow \infty$.

In Fig. 1, we plot the temporal evolution of this occupation probability $P(x, t)$ for different values of the dimensionless diffusion coefficient $\mathcal{D}$. For $\mathcal{D}$ greater than a critical value $\mathcal{D}_{c}$, the probability distribution is unimodal for all times. However, for $\mathcal{D}<\mathcal{D}_{c}(\approx 0.175)$, the occupation probability evolves from a unimodal distribution at short times, to a multimodal distribution at intermediate times and finally back to a unimodal distribution at long times. This non-trivial behavior of $P(x, t)$ for small $\mathcal{D}$ arises from the competition between the stochastic flipping of particle states (at rate $\gamma$ ) and translational diffusion. For a small $\mathcal{D}$, since $P_{ \pm}(x, 0)=\delta(x) / 2$, the RTPs in the $+(-)$ states move to the right (left) in an almost ballistic manner. This splits the initial unimodal distribution into a bimodal distribution with two symmetric peaks (see Fig. 1). While these two peaks are moving ballistically in opposite directions, they are also broadening because of the true diffusion term. As a result, at intermediate times when the tails of these two separated peaks meet at the centre, there again starts accumulation of particles (see Fig. 1(a)). This may lead to a central peak before the two ballistically moving side peaks disappear, which depends on the relative strengths of $\gamma$ and $\mathcal{D}$. Once developed, the central peak starts continuously broadening and on time scales much longer than the stochastic flipping rate $\gamma^{-1}$, the RTPs remix, leading to an effective diffusion constant as discussed above. As a result, the multimodal distribution 

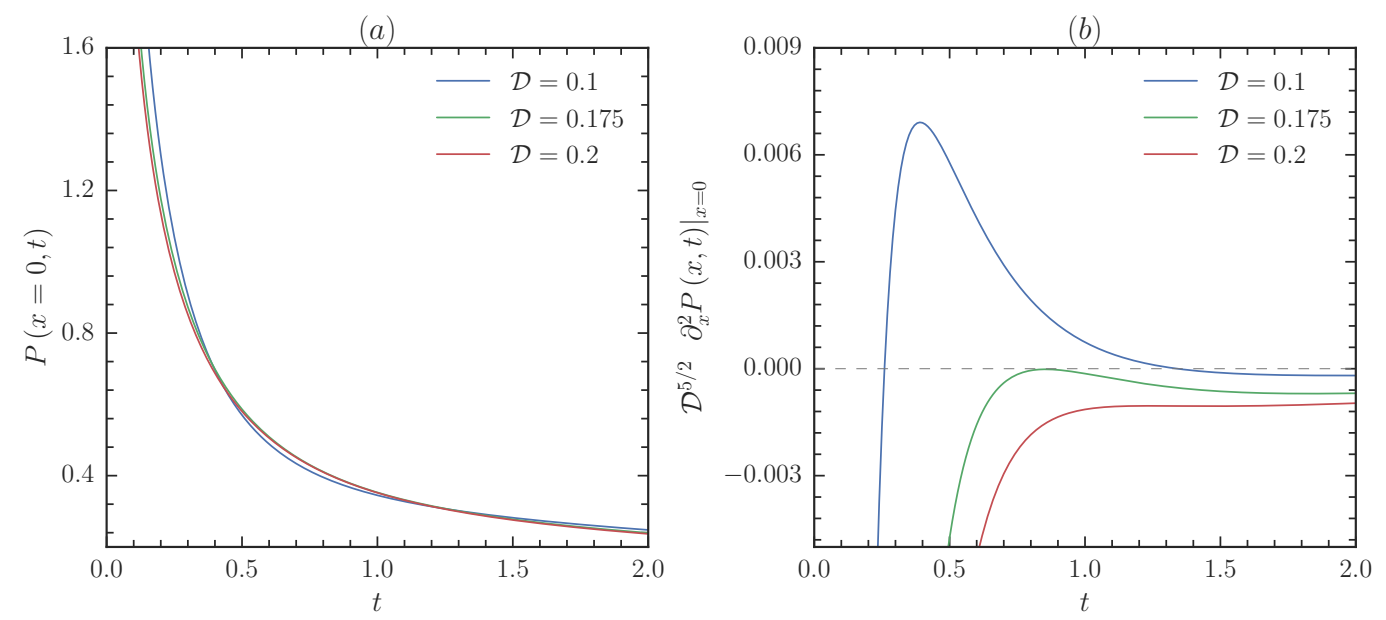

Figure 2. (a) The time-evolution of occupation probability $P(x, t)$ at $x=0$ for threedifferent values of the dimensionless diffusion coefficient $\mathcal{D}$. The occupation probability $P(0, t)$ is higher for smaller values of $\mathcal{D}$ at early-times, is lower at intermediate times and is again higher at long times. (b) The nature of the extremum of $P(x, t)$ at $x=0$ changes from being a maximum at earlier times to a minimum at intermediate times, and finally to a maximum at long times. This non-trivial behavior of the central extrema exists only for $\mathcal{D}<\mathcal{D}_{c} \approx 0.175$. For $\mathcal{D} \geq \mathcal{D}_{c}, P(x=0, t)$ is always a maxima for all times.

at intermediate times merges into a unimodal distribution, which as $t \rightarrow \infty$, converges to a Gaussian. On the other hand, for large $\mathcal{D}$, the split peaks of the two RTP states overlap to such an extent that the full distribution always remains unimodal. This behavior suggests that there exists a critical $\mathcal{D}$ where the effects of translational diffusion and stochastic flipping balance each other.

To understand this transition, we plot $P(x=0, t)$ for various $\mathcal{D}$ values in Fig. 2(a), and notice that the occupation probability at $x=0$ is higher for smaller $\mathcal{D}$ at short times as compared to that for larger $\mathcal{D}$. This then crosses over to a lower value at intermediate times and finally becomes larger at long times. Furthermore, to investigate the nature of the occupation probability at $x=0$, we plot the second derivative $\left.\partial_{x}^{2} P(x, t)\right|_{x=0}$. We find that for small $\mathcal{D}, P(x=0, t)$ has a maximum at short times, crosses over to a minimum at intermediate times, and finally crosses over to a maximum again at long times. However, for the critical value of $\mathcal{D}_{c} \approx 0.175$, these two crossover times merge, resulting in a unimodal distribution. For $\mathcal{D} \geq 0.175$, we find that $\left.\partial_{x}^{2} P(x, t)\right|_{x=0}$ is always negative and hence $P(x, t)$ is always unimodal.

In summary, we find that, in contrast to the Gaussian form for a Brownian particle, the probability distribution for an RTP can be multimodal depending on the value of the dimensionless diffusion coefficient $\mathcal{D}$. This diversity in the probability distribution also occurs in other systems in which the motion of a diffusing particle is influenced by an interplay with a convection field that changes sign [48]. 


\subsection{Bounded interval}

We now treat an RTP in the interval $x \in[-\ell, \ell]$. In this case, the probability distribution will reach a steady-state in the long time limit. For $v=0$, the particle performs pure Brownian motion and reaches a spatially uniform steady-state at long times. On the other hand if $\mathcal{D}=0$, the particle is subjected to only the dichotomous noise $\sigma(t)$. Here, the particle reaches a different steady-state in which, for any finite flipping rate, there is an accumulation of particles at the boundaries. However, for very large flipping rates, one regains a spatially uniform distribution with an diffusion effective coefficient $v^{2} / \gamma$. In the case where both $v$ and $\mathcal{D}$ are nonzero, we anticipate a steady-state which is intermediate to these two extreme cases. We first solve for the probability distribution in the steady-state and then we turn to the more complicated time-dependent solution.

In the steady-state, the dimensionless Fokker-Planck equations $(3 \mathrm{~b})$ reduce to

$$
\begin{aligned}
& \mathcal{D} \partial_{x}^{2} P_{+}-\partial_{x} P_{+}-P_{+}+P_{-}=0, \\
& \mathcal{D} \partial_{x}^{2} P_{-}+\partial_{x} P_{-}+P_{+}-P_{-}=0 .
\end{aligned}
$$

which we have to solve subject to the boundary conditions (5). The details of this calculation are given in Appendix A. The final result for the probability distribution is:

$$
P(x)=\left[\frac{\tanh \left(\frac{\sqrt{2 \mathcal{D}+1}}{\mathcal{D}} \ell\right)}{\sqrt{2 \mathcal{D}+1}}+2 \ell\right]^{-1}\left[\frac{\cosh \left(\frac{\sqrt{2 \mathcal{D}+1}}{\mathcal{D}} x\right)}{2 \mathcal{D} \cosh \left(\frac{\sqrt{2 \mathcal{D}+1}}{\mathcal{D}} \ell\right)}+1\right] .
$$

In Fig. 3, we compare (16) for the steady-state probability distribution $P(x)$ with results of simulation of the Langevin equation (1), and find nice agreement. We observe that probabilities are higher near the boundaries than at the center of the interval, in contrast to the uniform density which one would observe if there was no activity i.e. $v=0$. Such accumulation of active particles near the boundaries of a confined domain is quite generic and has been observed in experimental systems such as motile rods [49] and bacterial suspensions [50].

In the limit $\mathcal{D} \rightarrow 0$, the peaks near the boundaries become progressively sharper, and eventually become delta-function peaks. The full distribution is given by

$$
\left.P(x)\right|_{\mathcal{D} \rightarrow 0}=\frac{2+\delta(x-\ell)+\delta(x+\ell)}{2(1+2 \ell)} .
$$

We observe that the probability is uniform everywhere except for the delta function peaks at the boundaries. This $\mathcal{D} \rightarrow 0$ case has recently been considered in a similar context [40] and our method reproduces their results.

Let us now turn to the full time-dependent solution. We are interested in how the distribution $P(x, t)$ approaches the steady-state in the $t \rightarrow \infty$ limit. To this end, we have to solve the coupled time-dependent Fokker-Planck equations (3b) within the interval $x \in[-\ell, \ell]$, subject to the boundary conditions (5). For the time-dependent solution, we expand $P(x, t)$ in terms of the complete set of basis functions as

$$
\left(\begin{array}{l}
P_{+}(x, t) \\
P_{-}(x, t)
\end{array}\right)=\sum_{n} a_{n} e^{\lambda_{n} t}\left(\begin{array}{c}
\phi_{n}^{+}(x) \\
\phi_{n}^{-}(x)
\end{array}\right),
$$



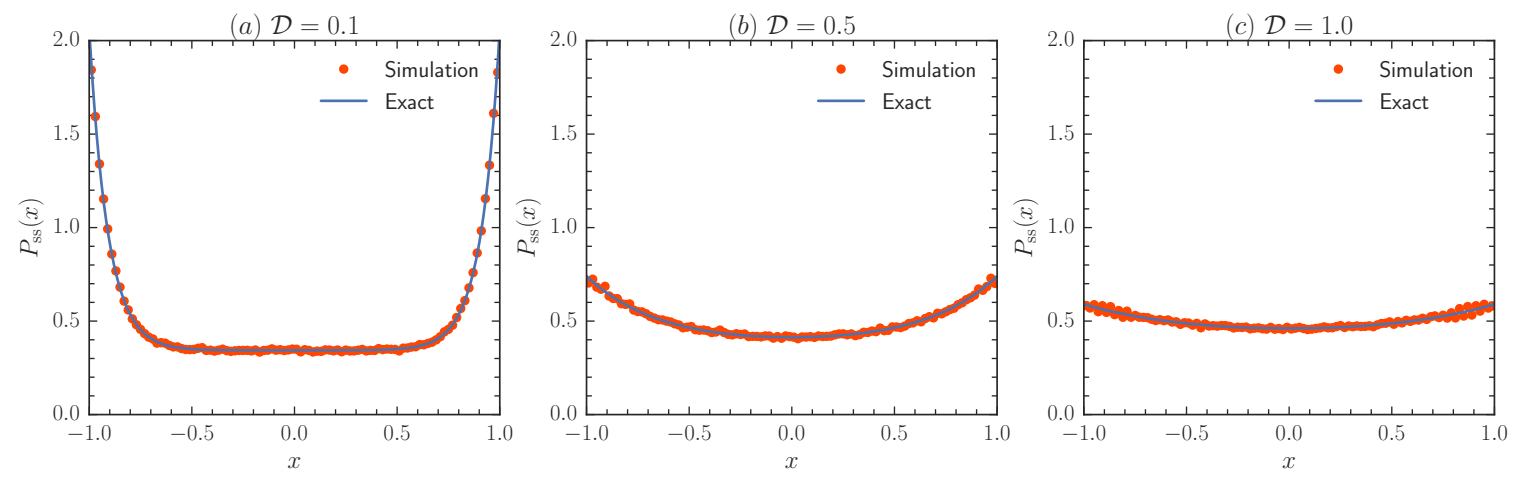

Figure 3. Comparison of the steady-state probability density equation (16) with explicit Langevin simulations for various $\mathcal{D}$. The histogram of the numerical simulation was constructed, at $t=5$, using $10^{6}$ different realizations of the stochastic process.

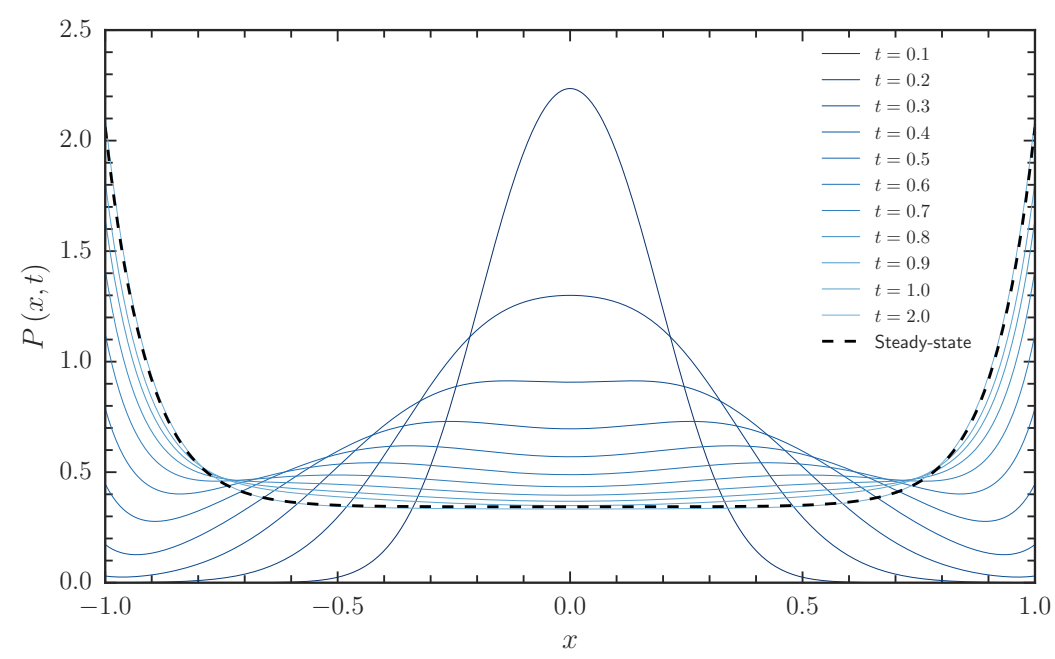

Figure 4. Time evolution of $P(x, t)$ in a interval obtained from solving the FokkerPlanck equations with appropriate boundary conditions. The diffusion constant was set to $\mathcal{D}=0.1$ and the data corresponds to an initial condition $P_{+}(x, 0)=P_{-}(x, 0)=$ $\delta(x) / 2$. It can be seen that at late times, the distribution converges to the exact steady-state distribution (16).

where $\lambda_{n}$ are the eigenvalues and the eigenfunctions $\left[\phi_{n}^{+}(x), \phi_{n}^{-}(x)\right]$ satisfy

$$
\begin{aligned}
& \mathcal{D} \partial_{x x} \phi_{n}^{+}-\partial_{x} \phi_{n}^{+}-\phi_{n}^{+}+\phi_{n}^{-}=\lambda_{n} \phi_{n}^{+}, \\
& \mathcal{D} \partial_{x x} \phi_{n}^{-}+\partial_{x} \phi_{n}^{-}+\phi_{n}^{+}-\phi_{n}^{-}=\lambda_{n} \phi_{n}^{-}
\end{aligned}
$$

subject to the boundary conditions (5). The coefficients $a_{n}$ are given in terms of the left eigenvectors $\left\langle\chi_{n}\right|=\left[\chi_{n}^{+}(x), \chi_{n}^{-}(x)\right]$ as

$$
a_{n}=\left\langle\chi_{n} \mid P(x, t=0)\right\rangle=\int_{-\ell}^{\ell} d x\left[\chi_{n}^{+}(x) P_{+}(x, t=0)+\chi_{n}^{-}(x) P_{-}(x, t=0)\right] .
$$

The left eigenvectors can be obtained as solutions of the adjoint Fokker-Planck operator. 
It can be shown that this has the same form as that in Eqs. (19), with the sign of the $\partial_{x}$ term changed, and with Neumann boundary conditions for both $\chi_{n}^{+}(x)$ and $\chi_{n}^{-}(x)$.

The details of calculating the eigenstates $\phi_{n}^{ \pm}(x)$ are given in Appendix B. Here we compare the time-evolution of the probability density obtained from a numerical solution of the Fokker-Planck equations (3b) using the boundary conditions (5), with the spectral expansion given by (18). The ground state eigenvalue $\lambda_{0}=0$, and the corresponding eigenstate (the steady-state) is known exactly and given by Eq. (16). In Fig. 4, we show the time-evolution of $P(x, t)$ obtained from a numerical solution of equations (3b). The unimodal to bimodal crossover discussed in the unbounded system can be seen in the figure. We also observe that our numerical solution converges to the exact steady-state. All the other eigenvalues $\lambda_{n}, n>0$ have to be found numerically from the zeros of the
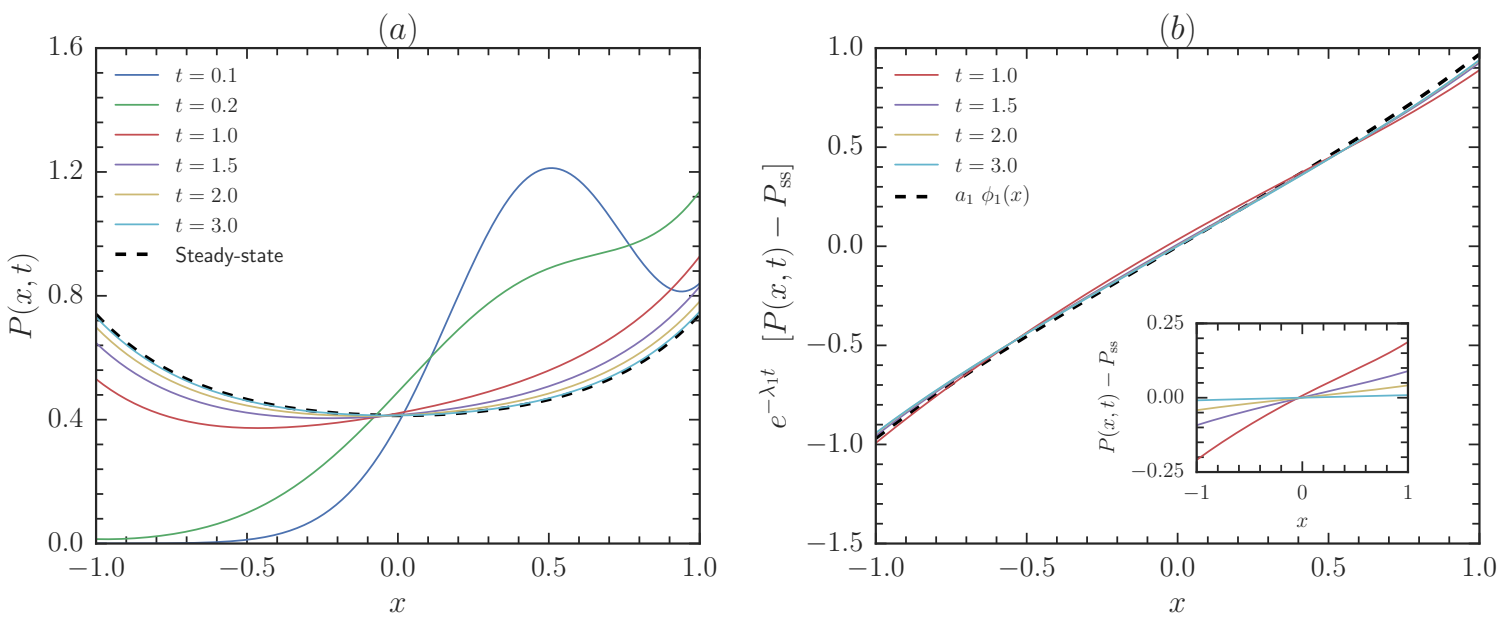

Figure 5. (a) Time evolution of $P(x, t)$ in an interval obtained from solving the Fokker-Planck equations with appropriate boundary conditions. The diffusion constant was set to $\mathcal{D}=0.5$ and the data corresponds to an initial condition $P_{+}(x, 0)=$ $P_{-}(x, 0)=\delta(x-1 / 2) / 2$. It can be seen that at long times, the distribution converges to the exact steady-state distribution. (b) Comparison of $\left(e^{-\lambda_{1} t}\left[P(x, t)-P_{S S}(x)\right]\right.$ with the eigenstate $\phi(x)$ corresponding to first excited state. The initial state here was chosen as $P_{ \pm}(x, t=0)=\delta(x-1 / 2) / 2$, same as in (a), which has $a_{1} \approx 0.7789$. The inset shows the unscaled data.

determinant of the matrix $M$ in (B.6). The long-time relaxation of the system to the steady-state would be determined by the eigenvalue with the largest non-zero real part, and the corresponding eigenfunction. As an illustrative example, we choose $\mathcal{D}=0.5$ and $\ell=1$, in which case the dominant eigenvalue is given by $\lambda_{1} \approx-1.55684$, while the 
corresponding eigenfunction is given by (B.5), with

$$
\begin{aligned}
& \left(\beta_{++}^{(1)}, \beta_{-+}^{(1)}, \beta_{+-}^{(1)}, \beta_{--}^{(1)}\right)=(1.66409,-1.66409,-0.998284 i, 0.998284 i) \\
& \left(\alpha_{++}^{(1)}, \alpha_{-+}^{(1)}, \alpha_{+-}^{(1)}, \alpha_{--}^{(1)}\right) \\
& =(-0.277351,-3.60554,-0.0585543-0.998284 i,-0.0585543+0.998284 i) \\
& \left(C_{++}^{(1)}, C_{-+}^{(1)}, C_{+-}^{(1)}, C_{--}^{(1)}\right) \\
& =(0.169039,0.0468831,-0.196156+0.184987 i,-0.196156-0.184987 i) .
\end{aligned}
$$

Thus we know the functions $\phi_{1}^{+}(x), \phi_{1}^{-}(x)$ explicitly, and in terms of these, we expect at long times

$$
P(x, t)=P_{+}(x, t)+P_{-}(x, t)=P_{S S}+a_{1} e^{\lambda_{1} t}\left[\phi_{1}^{+}(x)+\phi_{1}^{-}(x)\right]+\ldots .
$$

The parameter $a_{1}$ depends on initial conditions and can be obtained from the corresponding left eigenvector $\left\langle\chi_{1}\right|$ and we find $a_{1}=\langle\chi \mid P(t=0)\rangle=0.7789 \ldots$ In Fig. (5a), we plot the evolution of $P(x, t)$ obtained from a direct numerical solution of Eqs. (3b) and (5), starting from an initial condition $\delta(x-1 / 2)$. The plot in Fig. (5b) shows $P(x, t)-P_{S S}$ and compares this with the prediction from the first term in the spectral representation Eq. (21). It is seen that agreement is very good. In general, we find that eigenvalues can have imaginary parts (in which case they come in complex conjugate pairs) and so one can see oscillatory relaxation.

\section{First-Passage Properties}

In biological systems, we are often interested in the time required for a molecule diffusing in the interior of the cell to get adsorbed at the cell boundaries, as well as in the time required by a diffusing protein to find the correct binding sites. Similarly, in chemical reactions, an important quantity is the time spent by a reactive agent before it reaches catalytic boundaries. Hence it is important to compute quantities such as first-passage distributions, survival probabilities, and exit time distributions for the RTP. First-passage and survival probabilities of stochastic processes have been widely studied in the past (for reviews see [51, 52]). In the context of RTP in one dimension, with the position evolving via Eq. (1) but without the diffusion term, i.e., for $D=0$, first-passage properties have been studied before [47. More recently, the mean first-passage time between two points in space was computed for RTP (again for $D=0$ ) analytically [54]. For a RTP in one dimension, the mean first-passage time was recently measured numerically [55]. In this section we study the first-passage probability analytically for an RTP on a semi-infinite line and exit problem from a finite interval, in presence of telegraphic as well as the diffusive noise, i.e, when both terms in Eq. (1) are present. 


\subsection{First-passage on the semi-infinite line}

We are interested in the probability for an RTP, which starts from a point $x$ on the semiinfinite line with velocity \pm 1 , to arrive at the origin for the first time at time $t$. This quantity is directly related to the survival probability of the RTP in the same geometry in the presence of an absorbing boundary at $x=0$. Let $S_{+}(x, t)\left[S_{-}(x, t)\right]$ denote the probability that the RTP, starting initially at $x \geq 0$ with a positive [negative] velocity, survives being absorbed at the origin $x=0$ until time $t$, i.e., it does not cross the origin up to time $t$. Given the Langevin equation (1), it is convenient to write the backward Fokker-Planck equations for the evolution of $S_{ \pm}(x, t)$, where the initial position $x$ is treated as a variable [53]. Consider the evolution over the time window $[0, t+d t]$ and break it into two sub-intervals $[0, d t]$ and $[d t, t+d t]$. It follows from Eq. (1) that in a small time $d t$ following $t=0$ (i.e., during the first interval $[0, d t]$ ), the position of the particle evolves to a new position $x^{\prime}=x+v \sigma(0) d t+\sqrt{2 D} \eta(0) d t$, where $\sigma(0)$ and $\eta(0)$ are the initial noises. For the subsequent evolution in the time interval $[d t, t+d t]$, the new starting position is then $x^{\prime}$. Thus the survival probability satisfies the evolution equations

$$
\begin{aligned}
& S_{+}(x, t+d t)=(1-\gamma d t)\left\langle S_{+}(x+v d t+\sqrt{2 D} \eta(0) d t, t)\right\rangle+\gamma d t S_{-}(x, t), \\
& S_{-}(x, t+d t)=(1-\gamma d t)\left\langle S_{-}(x-v d t+\sqrt{2 D} \eta(0) d t, t)\right\rangle+\gamma d t S_{+}(x, t),
\end{aligned}
$$

where the \langle\rangle denotes the average over $\eta(0)$. Expanding in Taylor series for small $d t$, using the properties of $\eta(0)$ and taking $d t \rightarrow 0$ limit, one directly arrives at a pair of backward equations which read, in dimensionless units,

$$
\begin{aligned}
& \partial_{t} S_{+}(x, t)=-S_{+}+S_{-}+\partial_{x} S_{+}+\mathcal{D} \partial_{x}^{2} S_{+} \\
& \partial_{t} S_{-}(x, t)=S_{+}-S_{-}-\partial_{x} S_{-}+\mathcal{D} \partial_{x}^{2} S_{-} .
\end{aligned}
$$

These equations are valid for $x \geq 0$, with the initial conditions $S_{ \pm}(x, 0)=1$ for all $x>0$. In addition, we need to specify the boundary conditions. As the starting point $x \rightarrow \infty$, it is clear that $S_{ \pm}(x \rightarrow \infty, t)=1$, since the particle will surely not cross the origin in a finite time. In contrast, the boundary condition at $x=0$ is subtle: it depends on whether $\mathcal{D}=0$ or $\mathcal{D}>0$. Consider first the case $\mathcal{D}=0$, i.e., in absence of normal diffusion. In this case, if the particle starts at $x=0$ with a negative velocity, it will surely cross the origin in a finite time. Hence

$$
S_{-}(x=0, t)=0 \quad \text { when } \quad \mathcal{D}=0 .
$$

However, note that if the particle starts with a positive velocity, it can survive up to finite $t$, hence the boundary condition $S_{+}(0, t)$ is unspecified. We will see below that just one boundary condition in Eq. (24a) is sufficient to make the solution of Eqs. (23) unique. Under normal diffusion, it is well known that if a particle crosses the origin at some time, it recrosses it immediately infinitely often [52]. Hence, if the particle starts at the origin, no matter whether the initial velocity is positive or negative, it will surely 
cross zero within a short time $d t$, provided $\mathcal{D}>0$. This follows from the fact that in the $d t \rightarrow 0$ limit, the Brownian noise dominates over the drift term irrespective of its sign. Hence, in this case, we have the two boundary conditions

$$
S_{ \pm}(0, t)=0 \quad \text { when } \quad \mathcal{D}>0
$$

We will see later that indeed for $\mathcal{D}>0$, we will need both boundary conditions in Eq. (24b) to fix the solutions of Eq. (23) uniquely.

It is convenient to first take a Laplace transform, with respect to time $t$, of the pair of equations (23). Using the initial conditions $S_{ \pm}(x, 0)=1$, it is easy to see that the Laplace transforms satisfy

$$
\begin{aligned}
-1+s \tilde{S}_{+}(x, s) & =\mathcal{D} \partial_{x}^{2} \tilde{S}_{+}+\partial_{x} \tilde{S}_{+}-\tilde{S}_{+}+\tilde{S}_{-}, \\
-1+s \tilde{S}_{-}(x, s) & =\mathcal{D} \partial_{x}^{2} \tilde{S}_{-}-\partial_{x} \tilde{S}_{-}-\tilde{S}_{-}+\tilde{S}_{+}
\end{aligned}
$$

where $\tilde{S}_{ \pm}(x, s)=\int_{0}^{\infty} d t e^{-s t} S_{ \pm}(x, t)$ is the Laplace transform.

These equations can be made homogeneous by the shift: $\tilde{S}_{ \pm}(x, s)=1 / s+U_{ \pm}(x, s)$, where $U_{ \pm}$satisfy

$$
\begin{aligned}
& {\left[\mathcal{D} \partial_{x}^{2}+\partial_{x}-(1+s)\right] U_{+}(x, s)=-U_{-}(x, s),} \\
& {\left[\mathcal{D} \partial_{x}^{2}-\partial_{x}-(1+s)\right] U_{-}(x, s)=-U_{+}(x, s) .}
\end{aligned}
$$

Furthermore, by differentiating twice, one can write closed equations for $U_{+}$and $U_{-}$

$$
\begin{aligned}
& {\left[\mathcal{D} \partial_{x}^{2}-\partial_{x}-(1+s)\right]\left[\mathcal{D} \partial_{x}^{2}+\partial_{x}-(1+s)\right] U_{+}(x, s)=U_{+}(x, s),} \\
& {\left[\mathcal{D} \partial_{x}^{2}+\partial_{x}-(1+s)\right]\left[\mathcal{D} \partial_{x}^{2}-\partial_{x}-(1+s)\right] U_{-}(x, s)=U_{-}(x, s) .}
\end{aligned}
$$

Below, we first solve the simpler case $\mathcal{D}=0$, followed by the more complex $\mathcal{D}>0$ case.

4.1.1. The case $\mathcal{D}=0$. This particular case has been considered earlier with spacedependent transition rates, where only the mean first-passage time was computed [56]. Here we are interested in the full first-passage time distribution, which we can obtain using the above backward Fokker-Plank equation approach. For $\mathcal{D}=0$, Eqs. (27) are ordinary second-order differential equations with constant coefficients. Hence, we can try solutions of the form: $U_{ \pm}(x, s) \sim e^{-\lambda x}$. Substituting this in either of Eqs. (27), we find that $\lambda$ satisfies the quadratic equation, $(\lambda+1+s)(\lambda-1-s)=1$, which gives two roots: $\lambda(s)= \pm \sqrt{s^{2}+2 s}$. Obviously, the negative root is not admissible, since the solution must remain finite as $x \rightarrow \infty$. Retaining only the positive root, the general solutions of Eqs. (27) can be written as

$$
U_{+}(x, s)=B e^{-\lambda(s) x} ; \quad U_{-}(x, s)=A e^{-\lambda(s) x} \quad \text { where } \quad \lambda(s)=\sqrt{s^{2}+2 s} .
$$

The two unknown constants $B$ and $A$ are however related, as they must also satisfy the pair of first-order equations (26) (upon setting $\mathcal{D}=0$ ). This gives $B=A /[1+s+\lambda(s)]$. 
Hence, finally, using $\tilde{S}_{ \pm}(x, s)=1 / s+U_{ \pm}(x, s)$, we get

$$
\begin{aligned}
& \tilde{S}_{+}(x, s)=\frac{1}{s}+\frac{A}{1+s+\lambda(s)} e^{-\lambda(s) x}, \\
& \tilde{S}_{-}(x, s)=\frac{1}{s}+A e^{-\lambda(s) x},
\end{aligned}
$$

where $\lambda(s)=\sqrt{s^{2}+2 s}$. It remains to fix the only unknown constant $A$. This is done by using the boundary condition $\tilde{S}_{-}(0, s)=0$ which fixes $A=-1 / s$. Hence, we obtain the final solutions

$$
\begin{aligned}
& \tilde{S}_{+}(x, s)=\frac{1}{s}\left[1-\frac{1}{1+s+\lambda(s)} e^{-\lambda(s) x}\right], \\
& \tilde{S}_{-}(x, s)=\frac{1}{s}\left[1-e^{-\lambda(s) x}\right],
\end{aligned}
$$

with $\lambda(s)=\sqrt{s^{2}+2 s}$.

The first-passage time distribution is simply related to the survival probability via

$$
f_{+}(x, t)=-\partial_{t} S_{+}(x, t), \quad f_{-}(x, t)=-\partial_{t} S_{-}(x, t),
$$

or in Laplace variables

$$
\begin{aligned}
& \tilde{f}_{+}(x, s)=1-s \tilde{S}_{+}(x, s)=\frac{1}{(s+1+\lambda(s))} e^{-\lambda(s) x} \\
& \tilde{f}_{-}(x, s)=1-s \tilde{S}_{-}(x, s)=e^{-\lambda(s) x}
\end{aligned}
$$

where we recall $\lambda(s)=\sqrt{s^{2}+2 s}$.

It turns out that the Laplace transforms in Eqs. (32) can be exactly inverted. Before doing so, it is useful to extract the long-time asymptotics directly from the Laplace transforms in Eqs. (32), by considering the $s \rightarrow 0$ limit. A scaling limit then naturally emerges where $s \rightarrow 0, x \rightarrow \infty$ but with the product $x \sqrt{s}$ fixed. This corresponds, in the time domain, to the scaling limit $t \rightarrow \infty, x \rightarrow \infty$, but keeping the ratio $x / \sqrt{t}$ fixed. In this limit, $\lambda(s)=\sqrt{s^{2}+2 s} \rightarrow \sqrt{2 s}$ as $s \rightarrow 0$. Then, using the Laplace inversion

$$
\mathcal{L}^{-1}\left[e^{-a \sqrt{s}}\right]=\frac{a}{\sqrt{4 \pi t^{3}}} e^{-a^{2} / 4 t}
$$

we find that both $f_{ \pm}(x, t)$ converge, in the scaling limit, to the Holtsmark distribution

$$
f_{ \pm}(x, t) \rightarrow \frac{x}{\sqrt{4 \pi D_{0} t^{3}}} e^{-x^{2} / 4 D_{0} t} \quad \text { where } \quad D_{0}=\frac{1}{2}
$$

Now we recall that for a Brownian particle evolving via $d x / d t=\sqrt{2 D_{0}} \eta(t)$, the firstpassage probability $f(x, t)$ is given precisely [51] by the formula in Eq. (34). Hence, for our RTP that evolves via the telegraphic noise in Eq. (1) with $\mathcal{D}=0$, its first-passage probability in the scaling limit is equivalent to that for a normally diffusing particle with diffusion constant $D_{0}=1 / 2$. Indeed, this result is also consistent with our findings in 
Eq. 12, where we showed that, for $\mathcal{D}=0,\left\langle x^{2}(t)\right\rangle \rightarrow t$, which also corresponds to an effective normal diffusion at late times, with diffusion constant $D_{0}=1 / 2$.

To find the behavior of $f_{ \pm}(x, t)$ for finite $t$, we need to invert the Laplace transforms in Eqs. (32) exactly. Fortunately, this can be done using the following Laplace inversions

$$
\begin{aligned}
& \mathcal{L}^{-1}\left(\frac{e^{-x \lambda(s)}}{\lambda(s)[s+1+\lambda(s)]}\right)=e^{-t} \frac{\sqrt{t-x}}{\sqrt{t+x}} I_{1}\left(\sqrt{t^{2}-x^{2}}\right), \\
& \mathcal{L}^{-1}\left(\frac{e^{-x \lambda(s)}}{\lambda(s)}\right)=e^{-t} I_{0}\left(\sqrt{t^{2}-x^{2}}\right),
\end{aligned}
$$

where $I_{0,1}(t)$ are modified Bessel functions and $\lambda(s)=\sqrt{s^{2}+2 s}$. Taking the derivative with respect to $x$, we obtain

$$
\begin{aligned}
& f_{+}(x, t)=\frac{e^{-t}}{t+x}\left[x I_{0}\left(\sqrt{t^{2}-x^{2}}\right)+\frac{\sqrt{t-x}}{\sqrt{t+x}} I_{1}\left(\sqrt{t^{2}-x^{2}}\right)\right] \theta(t-x), \\
& f_{-}(x, t)=e^{-t} \frac{x}{\sqrt{t^{2}-x^{2}}} I_{1}\left(\sqrt{t^{2}-x^{2}}\right) \theta(t-x)+e^{-t} \delta(t-x) .
\end{aligned}
$$

These results match with those obtained by Orsingher [57] using a different approach.

In Fig. 6 we verify these results for the first-passage time distributions with simulations. For any given $x$, the large $t$ limit of (35) can be taken by using the asymptotic behavior $I_{1}(z) \rightarrow e^{z} / \sqrt{2 \pi z}$ as $z \rightarrow \infty$. This yields, as $t \rightarrow \infty$, for any $x$,

$$
f_{-}(x, t) \simeq \frac{1}{\sqrt{2 \pi}} \frac{x}{t^{3 / 2}} \quad \text { and } \quad f_{+}(x, t) \simeq \frac{1}{\sqrt{2 \pi}} \frac{x+1}{t^{3 / 2}} .
$$

While the tail of $f_{-}(x, t)$ behaves exactly as in the case of Brownian diffusion with a diffusion coefficient $D_{0}=1 / 2$ with a starting position $x$, the tail of $f_{+}(x, t)$ is equivalent to that in a Brownian diffusion with a starting position $x+1$. The extra length $1(=v / \gamma)$ is the average distance the RTP with a positive velocity moves before taking the first turn. In Fig. 6 we compare the asymptotic results of (36) with numerical simulations and find very good agreement. From (36), the large time behavior of the survival probabilities are given by

$$
S_{-}(x, t) \sim \frac{x}{\sqrt{t}} \quad \text { and } \quad S_{+}(x, t) \sim \frac{x+1}{\sqrt{t}} .
$$

In comparison with a particle with the negative starting velocity, a particle with the positive starting velocity has a higher probability of survival.

4.1.2. The case $\mathcal{D} \neq 0$. As explained in the beginning of this subsection, the survival probabilities $S_{ \pm}(x, t)$ satisfy the boundary conditions in Eq. (24b), i.e., in the Laplace domain, $\tilde{S}_{ \pm}(x=0, s)=0$. In this case, the shifted functions $U_{ \pm}(x, s)$ each satisfy a fourth-order ordinary differential equation with constant coefficients, as seen from Eqs. (27). Trying again a solution of the form: $U_{ \pm}(x, s) \sim e^{-\lambda x}$, we find that $\lambda$ has now 4 possible values that are the roots of the fourth-order polynomial

$$
\left[\mathcal{D} \lambda^{2}-\lambda-(1+s)\right]\left[\mathcal{D} \lambda^{2}+\lambda-(1+s)\right]=1 .
$$


(a)

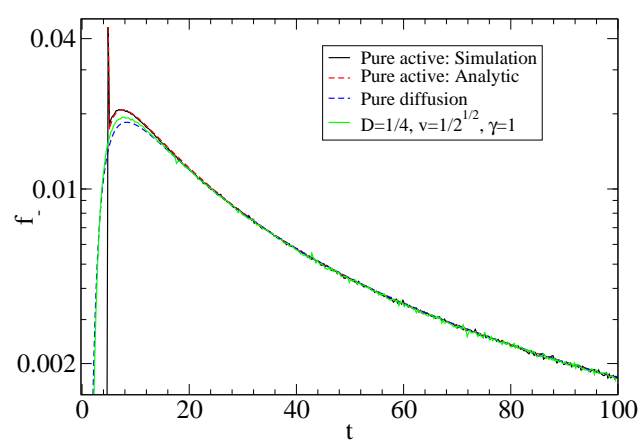

(b)

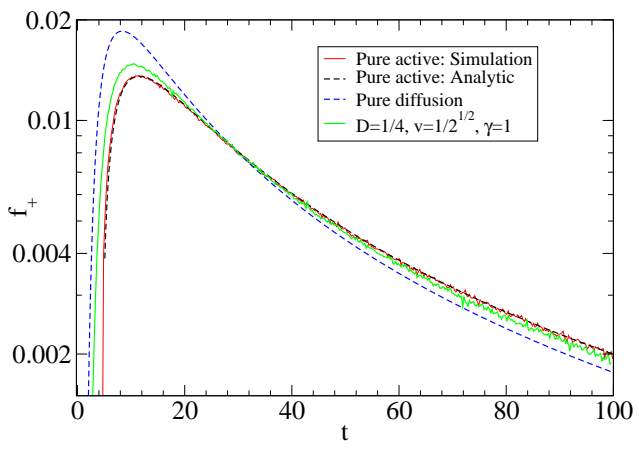

Figure 6. Comparison of the first-passage probability distributions $\gamma f_{ \pm}(x \gamma / v, t \gamma)$ from the exact results in (35) with with direct simulations of (1), with $D \equiv v^{2} \mathcal{D} /(2 \gamma)=$ $0, v=1, \gamma=1$ (pure active process). The starting point is taken to be $x=5$. The colored points correspond to simulation results while the black solid lines correspond to the exact result. Note that $\gamma f_{-}(x \gamma / v, t \gamma)$ has a $\delta$-function peak at $t=x$ corresponding to particles which reach the origin without any scattering. For comparison we also plot results for the pure diffusion case (with $D=1 / 2, v=0, \gamma=0$ ) and a mixed case. For the mixed case, the parameters are chosen as $D=1 / 4, v=1 / 2^{1 / 2}, \gamma=1$ so that the asymptotic effective diffusion constant is still $D+v^{2} /(2 \gamma)=1 / 2$

There are 4 solutions given by $\pm \lambda_{1}(s)$ and $\pm \lambda_{2}(s)$ where

$$
\begin{aligned}
& \lambda_{1}(s)=\left[\frac{1+\mathcal{D}(1+s)-\sqrt{1+4 \mathcal{D}(1+s)+4 \mathcal{D}^{2}}}{2 \mathcal{D}^{2}}\right]^{1 / 2}, \\
& \lambda_{2}(s)=\left[\frac{1+\mathcal{D}(1+s)+\sqrt{1+4 \mathcal{D}(1+s)+4 \mathcal{D}^{2}}}{2 \mathcal{D}^{2}}\right]^{1 / 2} .
\end{aligned}
$$

Evidently, $\lambda_{1}(s)<\lambda_{2}(s)$. Again discarding the negative roots $-\lambda_{1}(s)$ and $-\lambda_{2}(s)$ (since the solution cannot diverge as $x \rightarrow \infty)$, the general solutions of Eqs. (27) can be written as

$$
\begin{aligned}
& U_{+}(x, s)=B_{1} e^{-\lambda_{1}(s) x}+B_{2} e^{-\lambda_{2}(s) x}, \\
& U_{-}(x, s)=A_{1} e^{-\lambda_{1}(s) x}+A_{2} e^{-\lambda_{2}(s) x},
\end{aligned}
$$

where $\lambda_{1,2}(s)$ are given in Eqs. (39). However, these solutions must also satisfy the individual second-order equations (26). This indicates that $A_{1}, A_{2}$ are related to $B_{1}$ and $B_{2}$. Indeed, by substituting these solutions in Eqs. (26) gives the following relations

$$
\begin{aligned}
& B_{1}=-\frac{A_{1}}{\mathcal{D} \lambda_{1}^{2}-\lambda_{1}-(1+s)}=-A_{1}\left(\mathcal{D} \lambda_{1}^{2}+\lambda_{1}-(1+s)\right), \\
& B_{2}=-\frac{A_{2}}{\mathcal{D} \lambda_{2}^{2}-\lambda_{2}-(1+s)}=-A_{2}\left(\mathcal{D} \lambda_{2}^{2}+\lambda_{2}-(1+s)\right) .
\end{aligned}
$$

Note that we have used Eq. (38) to obtain the last two relations. 
Hence, the solutions for the survival probabilities are given by

$$
\begin{aligned}
& \tilde{S}_{+}(x, s)=\frac{1}{s}+B_{1} e^{-\lambda_{1} x}+B_{2} e^{-\lambda_{2} x}, \\
& \tilde{S}_{-}(x, s)=\frac{1}{s}+A_{1} e^{-\lambda_{1} x}+A_{2} e^{-\lambda_{2} x}
\end{aligned}
$$

where $B_{1}, B_{2}$ are related to $A_{1}$ and $A_{2}$ via Eqs. (41). We are still left with two unknown constants $A_{1}$ and $A_{2}$. To fix them, we use the two boundary conditions: $\tilde{S}_{ \pm}(x=0, s)=0$. This gives two linear equations for $A_{1}$ and $A_{2}$ whose solution is

$$
\begin{aligned}
& A_{1}=-\frac{1}{s} \frac{\mathcal{D} \lambda_{2}^{2}+\lambda_{2}-s}{\left[\mathcal{D}\left(\lambda_{2}^{2}-\lambda_{1}^{2}\right)+\lambda_{2}-\lambda_{1}\right]}, \\
& A_{2}=\frac{1}{s} \frac{\mathcal{D} \lambda_{1}^{2}+\lambda_{1}-s}{\left[\mathcal{D}\left(\lambda_{2}^{2}-\lambda_{1}^{2}\right)+\lambda_{2}-\lambda_{1}\right]} .
\end{aligned}
$$

This then uniquely determines the solutions for the survival probabilities $\tilde{S}_{ \pm}(x, s)$. The corresponding first-passage probabilities are given by

$$
\begin{aligned}
& \tilde{f}_{+}(x, s)=1-s \tilde{S}_{+}(x, s)=-s\left[B_{1} e^{-\lambda_{1} x}+B_{2} e^{-\lambda_{2} x}\right], \\
& \tilde{f}_{-}(x, s)=1-s \tilde{S}_{-}(x, s)=-s\left[A_{1} e^{-\lambda_{1} x}+A_{2} e^{-\lambda_{2} x}\right],
\end{aligned}
$$

where the constants $A_{1}, A_{2}, B_{1}$ and $B_{2}$ are determined explicitly above and $\lambda_{1,2}(s)$ are given in Eqs. (39).

The first nontrivial check is the limit $\mathcal{D} \rightarrow 0$. In this limit, it is easy to verify, from Eqs. (39), that

$$
\lambda_{1}(s) \rightarrow \lambda(s)=\sqrt{s^{2}+2 s}, \quad \text { and } \quad \lambda_{2}(s) \rightarrow \frac{1}{\mathcal{D}} \rightarrow \infty .
$$

In addition, one finds that as $\mathcal{D} \rightarrow 0$,

$$
A_{1} \rightarrow-\frac{1}{s}, \quad \text { and } \quad A_{2} \rightarrow 0
$$

and consequently

$$
B_{1} \rightarrow-\frac{1}{s\left(1+s+\sqrt{s^{2}+2 s}\right)}, \quad \text { and } \quad B_{2} \rightarrow 0 .
$$

We therefore recover the $\mathcal{D}=0$ results in Eqs. (32).

We now turn to the long-time asymptotic solutions of Eqs. (44) for arbitrary $\mathcal{D}$. Hence we consider the $s \rightarrow 0$ limit, with finite $\mathcal{D}$. In this limit, it is easy to check that, to leading order for small $s$

$$
\lambda_{1}(s) \rightarrow\left[\frac{2}{1+2 \mathcal{D}} s\right]^{1 / 2}, \quad \text { and } \quad \lambda_{2}(s) \rightarrow \frac{\sqrt{1+2 \mathcal{D}}}{\mathcal{D}} .
$$

Similarly, one can check that to leading order for small $s$

$$
s A_{1} \rightarrow-1, \quad \text { and } \quad s A_{2} \rightarrow O(\sqrt{s}),
$$


and consequently

$$
s B_{1} \rightarrow-1 \quad \text { and } \quad s B_{2} \rightarrow O(\sqrt{s}) .
$$

Substituting these results together in Eqs. (44), we find that in the scaling limit $(s \rightarrow 0$, $x \rightarrow \infty$ with the product $x \sqrt{s}$ fixed)

$$
\tilde{f}_{ \pm}(x, s) \rightarrow \exp \left[-\sqrt{\frac{2 s}{1+2 \mathcal{D}}} x\right]
$$

Upon inverting the Laplace transform using Eq. (33), we obtain our final results in the scaling limit

$$
f_{ \pm}(x, t) \rightarrow \frac{x}{\sqrt{4 \pi D_{1} t^{3}}} e^{-x^{2} / 4 D_{1} t}, \quad \text { where } \quad D_{1}=\mathcal{D}+\frac{1}{2}
$$

This result is precisely the same as the first-passage time density of an ordinary Brownian motion with diffusion constant $D_{1}=\mathcal{D}+1 / 2$. Note that for $D_{1} \rightarrow D_{0}=1 / 2$ as $\mathcal{D} \rightarrow 0$. Moreover, this effective diffusion constant $D_{1}=\mathcal{D}+1 / 2$ is consistent with our result $\left\langle x^{2}(t)\right\rangle \rightarrow(2 \mathcal{D}+1) t$ in Eq. 12 .

Unfortunately, unlike in the $\mathcal{D}=0$ case, for nonzero $\mathcal{D}$, we are not able to obtain the finite time result for $f_{ \pm}(x, t)$ explicitly, due to the fact that the Laplace transforms are difficult to invert.

\subsection{Exit probabilities and exit times in the finite interval}

We now investigate an RTP on a finite interval and address two questions: (a) the probability for the particle, which starts at $x$, to eventually reach either of the boundaries, and (b) the mean time for the particle to exit the interval by either of the boundaries. Let $E_{+}(x)\left(E_{-}(x)\right)$ denote the exit probabilities, namely the probability for a particle that starts at $x$ with velocity $+1(-1)$ exits through the boundary at $x=-\ell$. By comparison, the exit probability to $x=-\ell$ for isotropic diffusion, $E(x)$, is simply

$\frac{1}{2}\left(1-\frac{x}{\ell}\right)$; that is, the exit probability decreases linearly with the initial distance from the left edge.

It is easily seen that these hitting probabilities obey the backward equations 51

$$
\begin{aligned}
& \mathcal{D} \partial_{x}^{2} E_{+}+\partial_{x} E_{+}-\left(E_{+}-E_{-}\right)=0, \\
& \mathcal{D} \partial_{x}^{2} E_{-}-\partial_{x} E_{-}+\left(E_{+}-E_{-}\right)=0,
\end{aligned}
$$

subject to the appropriate boundary conditions, which are $E_{ \pm}(-\ell)=1$ and $E_{ \pm}(\ell)=0$. These boundary conditions fix the constants in $E_{ \pm}$and thus the problem is formally solved. The calculation is conceptually straightforward but tedious, and the details were performed by Mathematica. The basic steps and the final expressions for the exit probabilities are given by C.5 in Appendix C.

Fig. 7 shows the exit probabilities $E_{ \pm}(x)$ for representative values of the dimensionless diffusion coefficient $\mathcal{D}$. As one expects, for $\mathcal{D} \gg 1$, the exit probabilities 

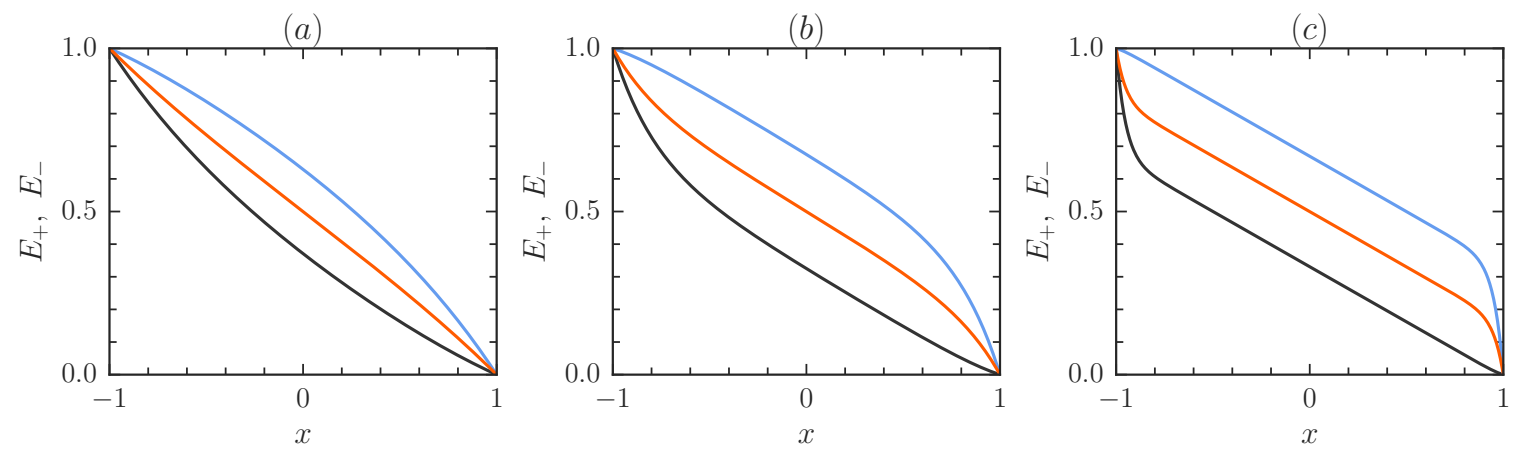

Figure 7. The exit probabilities $E_{+}(x)$ (black) and $E_{-}(x)$ (blue), and their average (orange), as a function of $x$ on an interval of scaled length $\ell=1$ for a particle with various diffusion (scaled) coefficient (a) $\mathcal{D}=1.0$, (b) $\mathcal{D}=0.25$, and (c) $\mathcal{D}=0.05$.
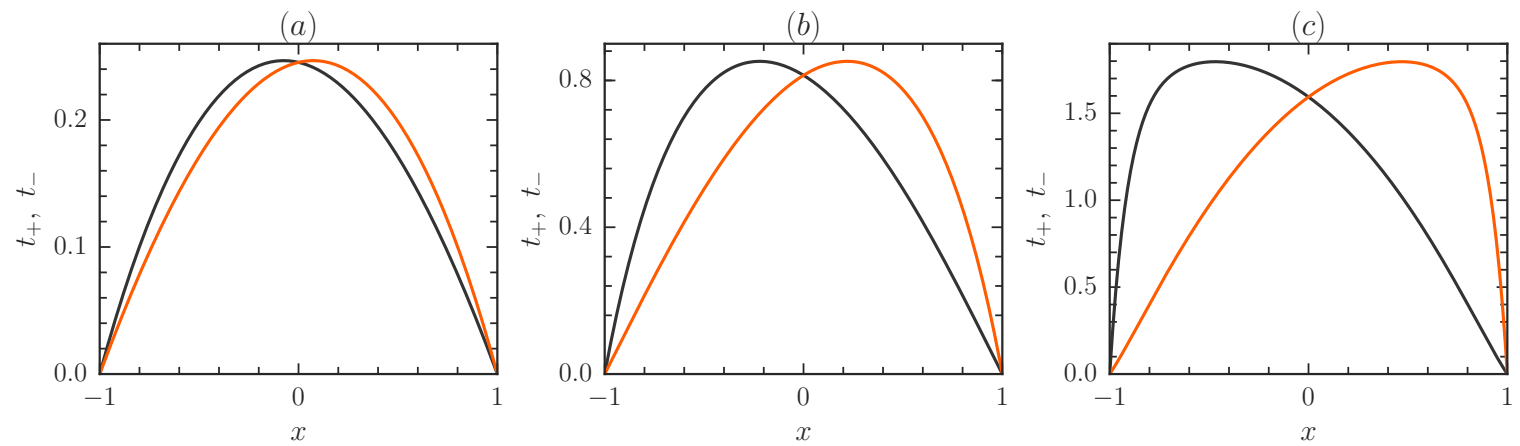

Figure 8. The unconditional exit times $t_{+}(x)$ (black) and $t_{-}(x)$ (orange) to either side of the interval as a function of $x$ for an interval of scaled length $\ell=1$ for a particle with various diffusion (scaled) coefficient (a) $\mathcal{D}=1.0$, (b) $\mathcal{D}=0.5$, and (c) $\mathcal{D}=0.1$.

are close to the isotropic random-walk form $\frac{1}{2}\left(1-\frac{x}{\ell}\right)$. However, for $\mathcal{D} \ll 1, E_{+}$and $E_{-}$become very distinct. Moreover, the exit probability $E_{+}$decreases much more rapidly with $x$ than $\left(1-\frac{x}{\ell}\right) / 2$, while $E_{-}$decreases much more slowly. Notice also that $\mathcal{E}(x) \equiv \frac{1}{2}\left[E_{+}(x)+E_{-}(x)\right] \neq \frac{1}{2}\left(1-\frac{x}{\ell}\right)$. That is, the exit probability, averaged over the two velocity states deviates significantly from the corresponding exit probability for unbiased diffusion.

Let us now turn to the exit times. Let $t_{+}(x, t)\left(t_{-}(x, t)\right)$ be the mean first-passage time (to either boundary) for a particle that is at $x$ and is also in the $+(-)$ state. Again using the formalism given in [51], it is easily seen that these exit times obey the backward equations

$$
\begin{aligned}
& \mathcal{D} \partial_{x}^{2} t_{+}+\partial_{x} t_{+}-\left(t_{+}-t_{-}\right)=-1, \\
& \mathcal{D} \partial_{x}^{2} t_{-}-\partial_{x} t_{-}+\left(t_{+}-t_{-}\right)=-1,
\end{aligned}
$$

with boundary conditions $t_{ \pm}( \pm \ell)=0$, which corresponds to the particle being immediately absorbed if it starts at either end of the interval. The solution to Eqs. (52) are obtained using the same approach as that given for Eqs. (51) (see Appendix D). While the resulting expressions for $t_{ \pm}$for the finite interval are too long to be displayed, 
the form of the first-passage times are easily visualized (Fig. 8). For small bias velocity the scaled diffusion constant $\mathcal{D}=D \gamma / v^{2}$ (see Sec. 2) is large. In this limit the diffusive part of the dynamics for both types of the particles dominates. As a result, $t_{+} \approx t_{-}$, and both $t_{+}$and $t_{-}$become very close to the exit probability for isotropic diffusion $t=\left(\ell^{2}-x^{2}\right) / 2 \mathcal{D}$. On the other hand, if $v$ is increased $\mathcal{D}$ is decreases and the active contribution to the motion dominates. In this limit the exit times $t_{+}$and $t_{-}$strongly deviate from each other.

\section{Summary}

We studied a one-dimensional model of run-and-tumble particles in the presence of an additional diffusion term. On the infinite line we find that an initial localized distribution of particles evolves to a Gaussian distribution at long times, with the diffusion constant renormalized by the active particle speed and the tumble rate, while at intermediate times the density distribution can have a multimodal structure.

In a finite domain with reflecting walls, we found that the RTPs reach a steady-state with peaks in the density distributions at the boundaries, which is in agreement with earlier observations of particle accumulation at walls. We also studied the approach to the steady-state by examining the spectral structure of the corresponding FokkerPlanck operator. The eigenvalues of this operator appear as the zeros of a complicated determinant, and finding them is highly non-trivial, even numerically. We numerically evaluated the two eigenvalues with largest real parts. It is an interesting mathematical problem to find the full spectrum as well as the associated eigenvectors of the FokkerPlanck operator.

We also investigated the first-passage probability distribution of an RTP on the semi-infinite line and obtained an explicit closed form expression for the distribution in the limiting case of zero diffusion and in the more challenging case of non-zero diffusion. In a finite domain, we obtained exact results for the exit time probability and the mean exit time.

We believe that our results for non-interacting RTPs in one dimension will be informative for the study of models of other active particle systems in higher dimensions. Another possible extension of this work is to study active particles in external potentials and in the presence of mutual interactions. It will be interesting to verify some of our analytic observations in experimental systems such as vibrated granular systems and Janus particles [7].

\section{Acknowledgment}

KM acknowledges the S. N. Bhatt Memorial Excellence Fellowship Program 2016 at ICTS, and INSPIRE-SHE (awarded by DST, Government of India) for funding her research. VJ is supported by a post-doctoral fellowship in the Max Planck partner group at ICTS AD, AK, SNM and SS acknowledge support from the Indo-French Centre 
for the promotion of advanced research (IFCPAR) under Project No. 5604-2. AD, AK, SNM and SS also acknowledge the large deviation theory program at ICTS (code: ICTS/Prog-ldt/2017/8) during which many discussions were held. SNM wishes to thank U. Basu. M.R. Evans, A. Rosso, and G. Schehr for useful discussions, and acknowledges a Simon foundation grant from ICTS. KVK's research is supported by the Department of Biotechnology, India, through a Ramalingaswami reentry fellowship and by the Max Planck Society and the Department of Science and Technology, India, through a Max Planck Partner Group at ICTS-TIFR. SR acknowledges support from grants DMR1608211 and DMR-1623243 from the National Science Foundation and from the ICTS for supporting his participation in the Bangalore school on statistical physics - VIII (code: ICTS/Prog-bssp/2017/06). He also thanks Uttam Bhat for many helpful discussions.

\section{References}

[1] Ramaswamy S 2017 Journal of Statistical Mechanics: Theory and Experiment 2017054002

[2] Prost J, Jülicher F and Joanny J F 2015 Nature Physics 11 111-117

[3] Marchetti M C, Joanny J F, Ramaswamy S, Liverpool T B, Prost J, Rao M and Simha R A 2013 Reviews of Modern Physics 85 1143-1189

[4] Berg H C and A B D 1972 Nature 239500

[5] Berg H and M P E 1977 Biophys. J. 20193

[6] Devreotes P N and Zigmond S H 1988 Annu. Rev. Cell Biol. 4649

[7] Bechinger C, Di Leonardo R, Löwen H, Reichhardt C, Volpe G and Volpe G 2016 Reviews of Modern Physics $\mathbf{8 8} 045006$

[8] Cates M E and Tailleur J 2013 EPL (Europhysics Letters) 10120010

[9] Tailleur J and Cates M E 2009 EPL (Europhysics Letters) 8660002

[10] Enculescu M and Stark H 2011 Phys. Rev. Lett. 107058301

[11] Lee C F 2013 New Journal of Physics 15055007

[12] Fily Y, Baskaran A and Hagan M F 2014 Soft Matter 105609

[13] Szamel G 2014 Physical Review E 90012111

[14] Solon A P, Cates M E and Tailleur J 2015 EPJST 2241231

[15] Vachier J and Mazza M G 2017 arXiv:1709.07488

[16] Hermann S and Schmidt M 2017 arXiv:1712.08553

[17] Romanczuk P, Bär M, Ebeling W, Lindner B and Schimansky-Geier L 2012 The European Physical Journal Special Topics 202 1-162

[18] Slowman A B, Evans M R and Blythe R A 2016 Phys. Rev. Lett. 116(21) 218101

[19] Slowman A B, Evans M R and Blythe R A 2017 Journal of Physics A: Mathematical and Theoretical 50375601

[20] Stenhammar J, Wittkowski R, Marenduzzo D and Cates M E 2015 Physical Review Letters 114 018301

[21] Reichhardt C J O and Reichhardt C 2017 Annual Review of Condensed Matter Physics 8 51-75

[22] Cates M E and Tailleur J 2015 Annual Review of Condensed Matter Physics 6 219-244

[23] Redner G S, Hagan M F and Baskaran A 2013 Phys. Rev. Lett. 110(5) 055701

[24] Stenhammar J, Tiribocchi A, Allen R J, Marenduzzo D and Cates M E 2013 Physical Review Letters 111145702

[25] Patch A, Yllanes D and Marchetti M C 2017 Physical Review E 95012601

[26] Solon A P, Fily Y, Baskaran A, Cates M E, Kafri Y, Kardar M and Tailleur J 2015 Nature Physics $11673-678$

[27] Solon A P, Stenhammar J, Wittkowski R, Kardar M, Kafri Y, Cates M E and Tailleur J 2015 Physical Review Letters 114198301 
[28] Junot G, Briand G, Ledesma-Alonso R and Dauchot O 2017 Physical Review Letters 119028002

[29] Fox R F 1986 Physical Review A 344525

[30] Hänggi P and Jung P 1995 Advances in chemical physics 89 239-326

[31] Dhar A and Chaudhuri D 2002 Physical Review Letters 89065502

[32] Samuel J and Sinha S 2002 Physical Review E 66050801

[33] Kurzthaler C, Leitmann S and Franosch T 2016 Scientific Reports 636702

[34] Tailleur J and Cates M 2008 Physical Review Letters 100218103

[35] Argun A, Moradi A R, Pince E, Bagci G B, Imparato A and Volpe G 2016 Physical Review E 94 062150

[36] Das S, Gompper G and Winkler R G 2017, New Journal of Physics

[37] Klein S, Appert-Rolland C and Evans M R 2016 Journal of Statistical Mechanics: Theory and Experiment 2016093206

[38] Maggi C, Marconi U M B, Gnan N and Leonardo R D 2015 Scientific Reports 510742

[39] Wagner C G, Hagan M F and Baskaran A 2017 Journal of Statistical Mechanics: Theory and Experiment 2017043203

[40] Angelani L 2017 Journal of Physics A: Mathematical and Theoretical 50325601

[41] Elgeti J and Gompper G 2015 EPL (Europhysics Letters) 10958003

[42] Taylor G I 1953 Proceedings of the Royal Society of London A: Mathematical, Physical and Engineering Sciences 219 186-203

[43] Aris R 1956 Proceedings of the Royal Society of London A: Mathematical, Physical and Engineering Sciences $23567-77$

[44] de Arcangelis L, Koplik J, Redner S and Wilkinson D 1986 Phys. Rev. Lett. 57(8) 996-999

[45] Koplik J, Redner S and Wilkinson D 1988 Phys. Rev. A 37(7) 2619-2636

[46] Whittaker E T 1910 A History of the Theories of Aether and Electricity from the Age of Descartes to the Close of the Nineteenth Century (Longmans, Green and Company)

[47] Weiss G H 2002 Physica A: Statistical Mechanics and its Applications 311 381-410

[48] Ben-Naim E, Redner S and ben Avraham D 1992 Phys. Rev. A 45(10) 7207-7213

[49] Antoine Bricard, Jean-Baptiste Caussin, Nicolas Desreumaux, Olivier Dauchot and Denis Bartolo 2013 Nature 503 95-98

[50] Di Leonardo R, Angelani L, Dell'Arciprete D, Ruocco G, Iebba V, Schippa S, Conte M P, Mecarini F, De Angelis F and Di Fabrizio E 2010 Proceedings of the National Academy of Sciences 107 9541-9545

[51] Redner S 2001 A Guide to First-Passage Processes (Cambridge University Press)

[52] Bray A J., Majumdar S N., and Schehr, G 2013 Adv. in Phys. 62225

[53] Majumdar S N 2005 Curr. Sci. 892076

[54] Angelani L, Di Lionardo R, and Paoluzzi M 2014 Euro. J. Phys. E 37, 59

[55] Scacchi A and Sharma A 2017 arXiv preprint arXiv:1708.05591

[56] Masoliver J, Porra J M and Weiss G H 1992 Physical Review A 452222

[57] Orsingher E 1995, Random Oper. and Stoch. Equ., 3, 9

\section{Appendix A. Steady-State Probability Distribution in the Interval}

We define $Q=P_{+}-P_{-}$and use $P=P_{+}+P_{-}$. With these definitions, 15 can be rewritten as:

$$
\begin{aligned}
\mathcal{D} \partial_{x}^{2} P-\partial_{x} Q & =0, \\
\mathcal{D} \partial_{x}^{2} Q-\partial_{x} P-2 Q & =0,
\end{aligned}
$$


and the boundary conditions (5) now read

$$
\begin{aligned}
& \left.\left(\mathcal{D} \partial_{x} Q-P\right)\right|_{x= \pm \ell}=0, \\
& \left.\left(\mathcal{D} \partial_{x} P-Q\right)\right|_{x= \pm \ell}=0 .
\end{aligned}
$$

Integrating the first of A.1 , we get $\mathcal{D} \partial_{x} P-Q+C_{1}=0$ where $C_{1}$ is an integration constant. From the second boundary condition in (A.2), we get $C_{1}=0$. Hence $\mathcal{D} \partial_{x} P(x)=Q(x)$ for all $x \in[-\ell, \ell]$, and substituting this into the second of A.1 leads to

$$
\mathcal{D} \partial_{x}^{2} Q-\left(2+\mathcal{D}^{-1}\right) Q=0
$$

This equation has the general solution

$$
Q=a e^{\mu x}+b e^{-\mu x}, \quad \text { with } \mu=\frac{\sqrt{2 \mathcal{D}+1}}{\mathcal{D}},
$$

and $a$ and $b$ are constants to be determined.

Once $Q(x)$ is known, $P(x)$ can be obtained by integrating $\mathcal{D} \partial_{x} P(x, t)=Q(x, t)$ :

$$
P(x)=\frac{1}{\mathcal{D} \mu}\left(a e^{\mu x}-b e^{-\mu x}\right)+C_{2},
$$

where $C_{2}$ is another integration constant. The three constants $a, b$ and $C_{2}$ can be obtained using the boundary conditions and the normalization condition. Substituting the solutions A.4 and A.5 into the first of $\mathrm{A}$ (A) gives

$$
\left(a e^{\mu \ell}-b e^{-\mu \ell}\right)=\frac{\mu}{2} C_{2}, \quad \text { and }\left(a e^{-\mu \ell}-b e^{\mu \ell}\right)=\frac{\mu}{2} C_{2},
$$

whose solution is

$$
a=\frac{\mu}{4 \cosh (\mu \ell)} C_{2} ; \quad \text { and } \quad b=-a .
$$

Finally, we invoke the normalization condition $\int_{-\ell}^{\ell} P(x) d x=1$ to obtain

$$
Q(x)=\frac{(2 \mathcal{D}+1)}{2 \mathcal{D}\left[\sinh \left(\frac{\sqrt{2 \mathcal{D}+1}}{\mathcal{D}} \ell\right)+2 \ell \sqrt{2 \mathcal{D}+1} \cosh \left(\frac{\sqrt{2 \mathcal{D}+1}}{\mathcal{D}} \ell\right)\right]} \sinh \left(\frac{\sqrt{2 \mathcal{D}+1}}{\mathcal{D}} x\right),
$$

and

$$
P(x)=\left[\frac{\tanh \left(\frac{\sqrt{2 \mathcal{D}+1}}{\mathcal{D}} \ell\right)}{\sqrt{2 \mathcal{D}+1}}+2 \ell\right]^{-1}\left[\frac{\cosh \left(\frac{\sqrt{2 \mathcal{D}+1}}{\mathcal{D}} x\right)}{2 \mathcal{D} \cosh \left(\frac{\sqrt{2 \mathcal{D}+1}}{\mathcal{D}} \ell\right)}+1\right] .
$$

The latter is 16 in the main text. 


\section{Appendix B. Time-Dependent Probability Distribution in the Interval}

We now construct the eigenstates $\phi_{n}^{ \pm}(x)$. First we try a solution of the form

$$
\left(\begin{array}{l}
\phi^{+}(x) \\
\phi^{-}(x)
\end{array}\right)=e^{\beta x}\left(\begin{array}{l}
r^{+} \\
r^{-}
\end{array}\right) \text {. }
$$

Inserting this form in (19), we get

$$
\left(\begin{array}{cc}
\mathcal{D} \beta^{2}-\beta-\lambda-1 & 1 \\
1 & \mathcal{D} \beta^{2}+\beta-\lambda-1
\end{array}\right)\left(\begin{array}{l}
r^{+} \\
r^{-}
\end{array}\right)=\left(\begin{array}{l}
0 \\
0
\end{array}\right) .
$$

To get non-zero solutions for $\bar{r}_{ \pm}$, we require the determinant of the matrix in the above equation to be zero: $\left(\mathcal{D} \beta^{2}-\lambda-1\right)^{2}-\left(\beta^{2}+1\right)=0$ which provides $\beta$ as function of $\lambda$. This is a fourth order equation in $\beta$ whose solutions are

$$
\beta_{\sigma \tau}(\lambda)=\sigma \sqrt{\frac{2 \mathcal{D}+1+2 \mathcal{D} \lambda+\tau \sqrt{(2 \mathcal{D}+1)^{2}+4 \mathcal{D} \lambda}}{2 \mathcal{D}^{2}}},
$$

where $\sigma= \pm 1$ and $\tau= \pm 1$. Corresponding to the resulting four values of $\beta$, we get the four corresponding solutions

$$
\left(\begin{array}{c}
r_{\sigma \tau}^{+} \\
r_{\sigma \tau}^{-}
\end{array}\right)=\left(\begin{array}{c}
1 \\
\alpha_{\sigma \tau}
\end{array}\right)
$$

where $\alpha_{\sigma \tau}=-\left(\mathcal{D} \beta_{\sigma \tau}^{2}-\beta_{\sigma \tau}-\lambda-1\right)$ and $\sigma=\tau= \pm 1$. We use these four states to construct the eigenstates $\left[\phi_{n}^{+}(x), \phi_{n}^{-}(x)\right]$ that satisfy the boundary conditions. Thus let

$$
\left(\begin{array}{c}
\phi_{n}^{+}(x, t) \\
\phi_{n}^{-}(x, t)
\end{array}\right)=\sum_{\sigma= \pm 1} \sum_{\tau= \pm 1} C_{n}^{\sigma \tau} e^{\beta_{\sigma \tau}^{(n)} x}\left(\begin{array}{c}
1 \\
\alpha_{\sigma \tau}
\end{array}\right)
$$

Substituting this solution into the required boundary conditions (5), and after some rearrangement, we get

$$
M\left(\begin{array}{l}
C_{n}^{++} \\
C_{n}^{+-} \\
C_{n}^{-+} \\
C_{n}^{--}
\end{array}\right)=0, \quad \text { where } \quad M=\left(\begin{array}{cccc}
\nu_{++}^{+-} & \nu_{+-}^{+-} & \nu_{-+}^{+-} & \nu_{--}^{+-} \\
\nu_{++}^{++} & \nu_{+-}^{++} & \nu_{-+}^{++} & \nu_{--}^{++} \\
\nu_{+-}^{--} & \nu_{+-}^{--} & \nu_{-+}^{--} & \nu_{--}^{--} \\
\nu_{++}^{-+} & \nu_{+-}^{-+} & \nu_{-+}^{-+} & \nu_{--}^{-+}
\end{array}\right)
$$

with $\nu_{\sigma \tau}^{r s}=e^{r \beta_{\sigma \tau} \ell}\left(\mathcal{D} \beta_{\sigma \tau}+s\right)$, and $\sigma, \tau, r, s$ allowed to take values \pm 1 . To get nonzero solutions for $C_{n}^{\sigma \tau}$, we require $\operatorname{det}(M)=0$. This equation has both real and imaginary parts and both have to be set to zero. This is possible only at certain values (in general complex) of $\lambda$ and these values then give us the required eigenvalue set $\left\{\lambda_{n}\right\}, n=0,1,2, \ldots$. We assume that the eigenvalues are ordered according to decreasing value of their real part. For each allowed $\lambda_{n}$ one can find the corresponding value of $\beta_{\sigma \tau}^{n}$ from $(\mathrm{B} .3)$. If the $\beta$ s are non-degenerate then the associated eigenvector 
$\left(C_{n}^{++}, C_{n}^{+-}, C_{n}^{-+}, C_{n}^{--}\right)^{T}$ can be obtained from (B.6). This then determines the eigenstates completely, up to a normalization constant.

We expect that there should be a real largest eigenvalue $\lambda_{0}=0$ corresponding to the steady-state and this was already determined, see (16). This solution can be recovered from our present approach but needs some extra care since for this case, $\beta_{ \pm-}=0$ and $\beta_{ \pm+}= \pm \sqrt{2 \mathcal{D}+1} / \mathcal{D}$. The two independent states corresponding to $\beta=0$ are given by

$$
\left(\begin{array}{l}
r_{+-}^{+} \\
r_{+-}^{-}
\end{array}\right)=\left(\begin{array}{l}
1 \\
1
\end{array}\right), \quad\left(\begin{array}{l}
r_{--}^{+} \\
r_{--}^{-}
\end{array}\right)=\left(\begin{array}{c}
x \\
1+x
\end{array}\right) .
$$

Taking a linear combination and imposing the boundary conditions leads us to the solution given in Eqs. (16) and (A.8).

\section{Appendix C. Solution for $E_{ \pm}(x)$ on the Finite Interval}

To solve Eqs. (51), we first define $S_{e}=E_{+}+E_{-}$and $\Delta_{e}=E_{+}-E_{-}$to recast (51) as

$$
\begin{aligned}
& \mathcal{D} S_{e}^{\prime \prime}+\Delta_{e}^{\prime}=0, \\
& \mathcal{D} \Delta_{e}^{\prime \prime}+S_{e}^{\prime}=2 \Delta_{e} .
\end{aligned}
$$

Differentiating the second of (C.1) and using the first to eliminate $S_{e}^{\prime \prime}$ gives $\Delta_{e}^{\prime \prime \prime}-\alpha^{2} \Delta_{e}^{\prime}=$ 0 , with

$$
\alpha^{2}=\frac{1}{\mathcal{D}^{2}}+\frac{2}{\mathcal{D}}
$$

The solution for $\delta_{e} \equiv \Delta_{e}^{\prime}$ is $\delta_{e}=A e^{\alpha x}+B e^{-\alpha x}$, where $A$ and $B$ are constants. Integrating once gives $\Delta_{e}$ and integrating $\mathcal{D} S_{e}^{\prime \prime}=-\Delta_{e}^{\prime}$ gives $S_{e}$. The final result is

$$
\begin{aligned}
& \Delta_{e}=\frac{A}{\alpha} e^{\alpha x}-\frac{B}{\alpha} e^{-\alpha x}+C, \\
& S_{e}=-\frac{A}{\mathcal{D} \alpha^{2}} e^{\alpha x}-\frac{B}{\mathcal{D} \alpha^{2}} e^{-\alpha x}+E x+F,
\end{aligned}
$$

where $C, E, F$ are constants. However, to satisfy the second of Eqs. (C.1), we must have $E=2 C$. Using this and finally solving for $E_{ \pm}$gives

$$
\begin{aligned}
& E_{+}(x)=\frac{1}{2} A e^{\alpha x}\left(\frac{1}{\alpha}-\frac{1}{\mathcal{D} \alpha^{2}}\right)-\frac{1}{2} B e^{-\alpha x}\left(\frac{1}{\alpha}+\frac{1}{\mathcal{D} \alpha^{2}}\right)+C x+\frac{1}{2}(F+C), \\
& E_{-}(x)=-\frac{1}{2} A e^{\alpha x}\left(\frac{1}{\alpha}+\frac{1}{\mathcal{D} \alpha^{2}}\right)+\frac{1}{2} B e^{-\alpha x}\left(\frac{1}{\alpha}-\frac{1}{\mathcal{D} \alpha^{2}}\right)+C x+\frac{1}{2}(F-C) .
\end{aligned}
$$

For exit via the left edge of the finite interval $[0, \ell]$, the appropriate boundary conditions are $E_{ \pm}(0)=1$ and $E_{ \pm}(\ell)=0$. Thus, from Eqs. (C.3), we need to solve

$$
\begin{aligned}
& A \gamma_{-}-B \gamma_{+}+\frac{1}{2}(F+C)=1 \\
& -A \gamma_{+}+B \gamma_{-}+\frac{1}{2}(F-C)=1 \\
& \quad A \gamma_{-} e^{\alpha \ell}-B \gamma_{+} e^{-\alpha \ell}+C \ell+\frac{1}{2}(F+C)=0 \\
& -A \gamma_{+} e^{\alpha \ell}+B \gamma_{-} e^{-\alpha \ell}+C \ell+\frac{1}{2}(F-C)=0
\end{aligned}
$$


where we have introduced

$$
\gamma_{ \pm}=\frac{1}{2}\left(\frac{1}{\alpha} \pm \frac{1}{\mathcal{D} \alpha^{2}}\right)
$$

Solving these four linear equations by Mathematica, substituting the coefficients $A, B, F$, and $C$ into C.3, and then performing some simplifications, the exit probabilities are:

$$
\begin{aligned}
& E_{+}(x)=\frac{e^{\alpha \ell}\left[(\ell-x)-\alpha \gamma_{-}\right]+\left[(\ell-x)+\alpha \gamma_{+}\right]+\gamma_{+} e^{\alpha(\ell-x)}+\gamma_{-} e^{\alpha \ell}}{e^{\alpha \ell}\left[\ell+\frac{1}{\mathcal{D} \alpha}\right]+\left[\ell-\frac{1}{\mathcal{D} \alpha}\right]}, \\
& E_{-}(x)=\frac{e^{\alpha \ell}\left[(\ell-x)+\alpha \gamma_{+}\right]+\left[(\ell-x)+\alpha \gamma_{-}\right]-\gamma_{-} e^{\alpha(\ell-x)}-\gamma_{+} e^{\alpha x}}{e^{\alpha \ell}\left[\ell+\frac{1}{\mathcal{D} \alpha}\right]+\left[\ell-\frac{1}{\mathcal{D} \alpha}\right]} .
\end{aligned}
$$

Some representative graphs of $E_{ \pm}(x)$ are given in Fig. 7.

\section{Appendix D. Solution for $t_{ \pm}(x)$ on the Finite Interval}

To solve (52), we again define $S_{t}=t_{+}+t_{-}, \Delta_{t}=t_{+}-t_{-}$to give

$$
\begin{aligned}
& \mathcal{D} S_{t}^{\prime \prime}+\Delta_{t}^{\prime}=-2, \\
& \mathcal{D} \Delta_{t}^{\prime \prime}+S_{t}^{\prime}=2 \Delta_{t} .
\end{aligned}
$$

We follow similar steps to those in Appendix C to obtain

$$
\begin{aligned}
& \Delta_{t}=\frac{A}{\alpha} e^{\alpha x}-\frac{B}{\alpha} e^{-\alpha x}-\frac{2 x}{(\mathcal{D} \alpha)^{2}}+C, \\
& S_{t}=-\frac{A}{\mathcal{D} \alpha^{2}} e^{\alpha x}-\frac{B}{\mathcal{D} \alpha^{2}} e^{-\alpha x}-\left(1-\frac{1}{(\mathcal{D} \alpha)^{2}}\right) \frac{x^{2}}{\mathcal{D}}+2 C x+F,
\end{aligned}
$$

where $C$ and $F$ are constants, and the additional terms compared to those in (C.2) stem from the additional inhomogeneous term in Eq. (D.1) compared to (C.1). The solutions for $t_{ \pm}$are

$$
\begin{aligned}
& t_{+}(x)=A \gamma_{-} e^{\alpha x}-B \gamma_{+} e^{-\alpha x}-\left(1-\frac{1}{(\mathcal{D} \alpha)^{2}}\right) \frac{x^{2}}{2 \mathcal{D}}+C x-\frac{x}{(\mathcal{D} \alpha)^{2}}+\frac{1}{2}(F+C) \\
& t_{-}(x)=-A \gamma_{+} e^{\alpha x}+B \gamma_{-} e^{-\alpha x}-\left(1-\frac{1}{(\mathcal{D} \alpha)^{2}}\right) \frac{x^{2}}{2 \mathcal{D}}+C x+\frac{x}{(\mathcal{D} \alpha)^{2}}+\frac{1}{2}(F-C)
\end{aligned}
$$

For the exit times $t_{ \pm}$in a finite interval of length $\ell$, the boundary conditions are $t_{ \pm}(0)=t_{ \pm}(\ell)=0$. Applying these boundary conditions to (D.3) fixes the constants $A, B, C$ and $F$, from which the results shown in Fig. 8 are obtained, again using Mathematica. 